JOURNAL OF

SYMPLECTIC GEOMETRY

Volume 7, Number 4, 475-502, 2009

\title{
QUANTIZATION OF SYMPLECTIC VECTOR SPACES OVER FINITE FIELDS
}

\author{
Shamgar Gurevich and Ronny Hadani
}

In this paper, we construct a quantization functor, associating a complex vector space $\mathcal{H}(V)$ to a finite-dimensional symplectic vector space $V$ over a finite field of odd characteristic. As a result, we obtain a canonical model for the Weil representation of the symplectic group $\mathrm{Sp}(V)$. The main new technical result is a proof of a stronger form of the Stone-von Neumann property for the Heisenberg group $H(V)$. Our result answers, for the case of the Heisenberg group, a question of Kazhdan about the possible existence of a canonical vector space attached to a coadjoint orbit of a general unipotent group over finite field.

\section{Introduction}

Quantization is a fundamental procedure in mathematics and in physics. Although it is widely used in both contexts, its precise nature remains to some extent unclear. From the physical side, quantization is the procedure by which one associates to a classical mechanical system its quantum counterpart. From the mathematical side, it seems that quantization is a way to construct interesting vector spaces out of symplectic manifolds, suggesting a method for constructing representations of the corresponding groups of symplectomorphisms $[\mathbf{1 5}, \mathbf{1 7}]$.

\subsection{Main results}

0.1.1. Quantization functor. In this paper, we construct a quantization functor

$$
\mathcal{H}: \text { Symp } \longrightarrow \text { Vect }
$$

where Symp denotes the (groupoid) category whose objects are finitedimensional symplectic vector spaces over the finite field $\mathbb{F}_{q}$, and morphisms are linear isomorphisms of symplectic vector spaces and Vect denotes the category of finite-dimensional complex vector spaces. 
0.1.2. Canonical model for the Weil representation. As a consequence, for a fixed symplectic vector space $V \in$ Symp, we obtain, by functoriality, a homomorphism

$$
\mathcal{H}: \mathrm{Sp}(V) \longrightarrow \mathrm{GL}(\mathcal{H}(V)),
$$

which is isomorphic to the Weil representation [19] of the group $\operatorname{Sp}(V)$ and we refer to it as the canonical model of the Weil representation.

Remark 0.1. The symplectic group acts on the canonical model $\mathcal{H}(V)$ in a natural way. This should be contrasted with the classical approach $[\mathbf{7}, \mathbf{1 3}, \mathbf{1 9}]$ in which one constructs a projective representation of the symplectic group (invoking the Stone-von Neumann (S-vN) theorem) and then deriving the linear action, implicitly, by invoking the fact that every projective representation of $\mathrm{Sp}(V)$ can be linearized.

0.1.3. Properties of the quantization functor. We show that the functor $\mathcal{H}$ is a monoidal functor, compatible with duality and with the operation of linear symplectic reduction. The last compatibility means that given $V \in$ Symp and a pair $I^{\circ}=\left(I, o_{I}\right)$, where $I \subset V$ is an isotropic subspace and $o_{I} \in \wedge^{\text {top }} I$ is a non-zero vector, ${ }^{1}$ there exists a natural isomorphism

$$
\mathcal{H}^{I}(V) \simeq \mathcal{H}\left(I^{\perp} / I\right)
$$

where $\mathcal{H}^{I}(V)$ stands for the subspace of $I$-invariant vectors in $\mathcal{H}(V)$ (an operation which will be made precise in the sequel) and $I^{\perp} / I \in$ Symp is the symplectic reduction of $V$ with respect to $I[\mathbf{3}]$. A particular situation is when $I=L$ is a Lagrangian subspace. In this situation, $L^{\perp} / L=0$ and (0.1) yields an isomorphism $\mathcal{H}^{L}(V) \simeq \mathcal{H}(0)=\mathbb{C}$, which associates to $1 \in \mathbb{C}$ a well-defined vector in $\mathcal{H}(V)$. This establishes a mechanism which associates to every pair $L^{\circ}=\left(L, o_{L}\right)$ a well-defined vector $v_{L^{\circ}} \in \mathcal{H}_{V}$. To the best of our knowledge (cf. $[\mathbf{1 1}, \mathbf{2 0}, \mathbf{2 1}])$, this kind of structure, which exists in the setting of the Weil representation of the group $\operatorname{Sp}(V)$, was not observed before.

0.1.4. The strong S-vN theorem. The main technical result of this paper is a proof $([\mathbf{8}, \mathbf{1 2}]$ unpublished) of a strong form of the $\mathrm{S}-\mathrm{vN}$ theorem for the Heisenberg group over $\mathbb{F}_{q}$ (see also the survey [9]). We supply two proofs; in the first proof we use only basic considerations from linear algebra and in the second proof we construct an algebra geometric object (an $\ell$-adic perverse Weil sheaf) which is interesting in its own sake and in particular implies the strong S-vN theorem.

We devote the rest of the introduction to an intuitive explanation of the main ideas and results of this paper.

\footnotetext{
${ }^{1}$ We will call the pair $I^{\circ}=\left(I, o_{I}\right)$ an oriented isotropic subspace.
} 


\subsection{The Heisenberg representation}

0.2.1. The Heisenberg group. Let $(V, \omega)$ be a $2 n$-dimensional symplectic vector space over the finite field $\mathbb{F}_{q}$. The vector space $V$ considered as an abelian group admits a non-trivial central extension called the Heisenberg group

$$
0 \longrightarrow \mathbb{F}_{q} \longrightarrow H(V) \longrightarrow V \longrightarrow 0 \text {. }
$$

Choosing a section $V \rightarrow H(V)$, the Heisenberg group can be presented as $H(V)=V \times \mathbb{F}_{q}$ with the center given by $Z=Z(H)=\left\{(0, z): z \in \mathbb{F}_{q}\right\}$. The symplectic group $\operatorname{Sp}(V)$ acts on $H(V)$ by group automorphisms via its tautological action on the $V$-coordinate.

0.2.2. The Heisenberg representation. The representation theory of the Heisenberg group is relatively simple. Given a non-trivial central character $\psi: Z \rightarrow \mathbb{C}^{\times}$, there exist a unique (up to isomorphism) irreducible representation

$$
\pi: H(V) \longrightarrow \mathrm{GL}(\mathcal{H})
$$

such that the center acts by $\psi$, i.e., $\pi_{\mid Z}=\psi \cdot \operatorname{Id}_{\mathcal{H}}$. This is the content of the celebrated S-vN theorem. The representation $\pi$ is referred to as the Heisenberg representation.

0.2.3. Models of the Heisenberg representation. The Heisenberg representation admits a family of models which are associated with oriented Lagrangian subspaces in $V[\mathbf{1}, \mathbf{1 8}]$. An oriented Lagrangian subspace is a pair $L^{\circ}=\left(L, o_{L}\right)$, where $L \subset V$ is a Lagrangian subspace and $o_{L} \in \wedge^{\text {top }} L$ is a non-zero vector. Every oriented Lagrangian $L^{\circ}$ is associated with a model

$$
\pi_{L^{\circ}}: H(V) \longrightarrow \operatorname{GL}\left(\mathcal{H}_{L^{\circ}}\right),
$$

where the vector space $\mathcal{H}_{L^{\circ}}$ is the space of functions $\mathbb{C}(L \backslash H(V), \psi)$, consisting of functions $f: H(V) \rightarrow \mathbb{C}$ such that $f(z \cdot l \cdot h)=\psi(z) f(h)$, for every $z \in Z, l \in L$ and the action $\pi_{L^{\circ}}$ is given by right translation.

Remark 0.2. The definition of the model $\left(\pi_{L^{\circ}}, H(V), \mathcal{H}_{L^{\circ}}\right)$ does not depend on the orientation $o_{L}$. The role of the orientation will appear later when we explain the relation between different models — the strong S-vN property.

0.3. The strong $\mathbf{S}-\mathbf{v N}$ property. The collection of models $\left\{\mathcal{H}_{L^{\circ}}\right\}$ can be thought of as a vector bundle $\mathfrak{H}$ on the set $\operatorname{OLag}(V)$ of oriented Lagrangians, with fibers $\mathfrak{H}_{\mid L^{\circ}}=\mathcal{H}_{L^{\circ}}$. The vector bundle $\mathfrak{H}$ is equipped with the following tautological structures:

- An action of the group $H(V)$ on each fiber.

- $\operatorname{An} \operatorname{Sp}(V)$-equivariant structure.

The content of the strong $\mathrm{S}-\mathrm{vN}$ theorem is that $\mathfrak{H}$ admits a canonical trivialization: 
Theorem 0.3 (Strong S-vN theorem). There exists a canonical system of intertwining morphisms $T_{M^{\circ}, L^{\circ}} \in \operatorname{Hom}_{H(V)}\left(\mathcal{H}_{L^{\circ}}, \mathcal{H}_{M^{\circ}}\right)$, for every $\left(M^{\circ}, L^{\circ}\right) \in \mathrm{OLag}(V)^{2}$, satisfying the following multiplicativity property:

$$
T_{N^{\circ}, M^{\circ} \circ} T_{M^{\circ}, L^{\circ}}=T_{N^{\circ}, L^{\circ}}
$$

for every $\left(N^{\circ}, M^{\circ}, L^{\circ}\right) \in \operatorname{OLag}(V)^{3}$.

Remark 0.4. The definition of the trivialization $\left\{T_{M^{\circ}, L^{\circ}}\right\}$ depends on the orientation structure.

0.3.1. Canonical vector space. The vector space $\mathcal{H}(V)$ is the space of "horizontal sections" of $\mathfrak{H}$

$$
\mathcal{H}(V)=\Gamma_{\text {hor }}(\operatorname{OLag}(V), \mathfrak{H}),
$$

where a vector in $\mathcal{H}(V)$ is a compatible system $\left(v_{L^{\circ}} \in \mathcal{H}_{L^{\circ}}: L^{\circ} \in \mathrm{OLag}(V)\right)$ such that $T_{M^{\circ}, L^{\circ}}\left(v_{L^{\circ}}\right)=v_{M^{\circ}}$, for every $\left(M^{\circ}, L^{\circ}\right) \in \operatorname{OLag}(V)^{2}$. The symplectic group $\operatorname{Sp}(V)$ acts on the vector space $\mathcal{H}(V)$ in an obvious manner. We denote this action by

$$
\rho_{V}: \mathrm{Sp}(V) \longrightarrow \mathrm{GL}(\mathcal{H}(V))
$$

and refer to it as the canonical model of the Weil representation.

We proceed to explain an algebra geometric construction of the trivialization $\left\{T_{M^{\circ}, L^{\circ}}\right\}$.

0.3.2. Canonical trivialization. The construction will be close in spirit to the procedure of "analytic continuation". Let $U_{2} \subset \operatorname{OLag}(V)^{2}$ denote the subset consisting of pairs $\left(M^{\circ}, L^{\circ}\right) \in \operatorname{OLag}(V)^{2}$ such that $M+L=V$. On the set $U_{2}$, the canonical intertwining morphisms are given by a uniform explicit formula - ansatz. The problem is how to extend this formula to the set of all pairs. An appealing way to do this is through the use of algebraic geometry.

0.3.3. Kernel functions. Every intertwining morphism $T_{M^{\circ}, L^{\circ}}$ can be uniquely presented by a function $K_{M^{\circ}, L^{\circ}} \in \mathbb{C}(M \backslash H(V) / L, \psi)$, which we refer to as the canonical kernel function. The collection of kernel functions $\left\{K_{M^{\circ}, L^{\circ}}:\left(M^{\circ}, L^{\circ}\right) \in U_{2}\right\}$ can be thought of as a single function $K_{O}$ on $O=U_{2} \times H(V)$ which is given by $K_{O}\left(M^{\circ}, L^{\circ},-\right)=K_{M^{\circ}, L^{\circ}}(-)$, for every $\left(M^{\circ}, L^{\circ}\right) \in U_{2}$. The problem now translates to the problem of extending the function $K_{O}$ to the set $X=\operatorname{OLag}(V)^{2} \times H(V)$. To this end, we invoke the procedure of geometrization.

0.4. Geometrization. A general ideology due to Grothendieck is that any meaningful set-theoretic object is governed by a more fundamental algebrageometric one. The procedure by which one translate from the set-theoretic setting to algebraic geometry is called geometrization, which is a formal procedure by which sets are replaced by algebraic varieties and functions are replaced by certain sheaf-theoretic objects. 
The precise setting consists of:

- A set $X=\mathbf{X}\left(\mathbb{F}_{q}\right)$ of rational points of an algebraic variety $\mathbf{X}$, defined over $\mathbb{F}_{q}$.

- A complex valued function $f \in \mathbb{C}(X)$ governed by an $\ell$-adic Weil sheaf $\mathcal{F}$.

The variety $\mathbf{X}$ is a space equipped with an automorphism $\mathrm{Fr}: \mathbf{X} \rightarrow \mathbf{X}$ (called Frobenius), such that the set $X$ is naturally identified with the set of fixed points $X=\mathbf{X}^{\mathrm{Fr}}$.

The sheaf $\mathcal{F}$ can be thought of as a vector bundle on the variety $\mathbf{X}$, equipped with an endomorphism $\theta: \mathcal{F} \rightarrow \mathcal{F}$ which lifts Fr.

The relation between the function $f$ and the sheaf $\mathcal{F}$ is called Grothendieck's sheaf-to-function correspondence: Given a point $x \in X$, the endomorphism $\theta$ restricts to an endomorphism $\theta_{x}: \mathcal{F}_{\mid x} \rightarrow \mathcal{F}_{\mid x}$ of the fiber $\mathcal{F}_{\mid x}$. The value of $f$ on the point $x$ is given by

$$
f(x)=f^{\mathcal{F}}(x)=\operatorname{Tr}\left(\theta_{x}: \mathcal{F}_{\mid x} \longrightarrow \mathcal{F}_{\mid x}\right) .
$$

0.5. Solution to the extension problem. The extension problem of the function $K_{O}$ fits nicely to the geometrization setting:

- The sets $O, X$ are sets of rational points of corresponding algebraic varieties $O=\mathbf{O}\left(\mathbb{F}_{q}\right)$ and $X=\mathbf{X}\left(\mathbb{F}_{q}\right)$. In addition, the imbedding $j: O \hookrightarrow X$ is induced from an open imbedding $j: \mathbf{O} \hookrightarrow \mathbf{X}$.

- The function $K_{O}$ comes from a Weil sheaf $\mathcal{K}_{\mathbf{O}}$ on the variety $\mathbf{O}$

$$
K_{O}=f^{\mathcal{K}_{\mathbf{O}}} \text {. }
$$

The extension problem is solved as follows: First extend the sheaf $\mathcal{K}_{\mathbf{O}}$ to a sheaf $\mathcal{K}$ on the variety $\mathbf{X}$ and then take the corresponding function $K=f^{\mathcal{K}}$, which establishes the desired extension. The reasoning behind this strategy is that in the realm of sheaves there exist several functorial operations of extension, probably the most interesting one is called perverse extension [2]. The sheaf $\mathcal{K}$ is defined as the perverse extension of $\mathcal{K}_{\mathbf{O}}$.

0.6. Structure of the paper. Apart from the introduction, the paper consists of two sections and two appendices.

In Section 1, the classical construction of the Weil representation is described. We begin with the definition of the Heisenberg group and the Heisenberg representation, then we briefly describe the classical construction of the Weil representation. In Section 2 we develop the framework of canonical vector spaces. Specifically, we introduce the canonical system of intertwining morphisms between different models of the Heisenberg representation and formulate the strong S-vN property of the Heisenberg representation (Theorem 2.5). Using Theorem 2.5, we construct a quantization functor $\mathcal{H}$. We finish this section by showing that $\mathcal{H}$ is a monoidal functor and it is compatible with duality and the operation of linear symplectic 
reduction. In Section 3, we construct a sheaf-theoretic counterpart of the canonical system of intertwining morphisms. Finally, in Appendix A, we provide proofs for all statements which appear in the body of the paper.

\section{The Weil representation}

1.1. The Heisenberg group. Let $(V, \omega)$ be a $2 n$-dimensional symplectic vector space over the finite field $\mathbb{F}_{q}$, where $q$ is odd. Considering $V$ as an abelian group, it admits a non-trivial central extension $H(V)$ called the Heisenberg group, namely

$$
0 \longrightarrow \mathbb{F}_{q} \longrightarrow H(V) \longrightarrow V \longrightarrow 0 .
$$

Concretely, the group $H(V)$ can be presented as the set $H(V)=V \times \mathbb{F}_{q}$ with the multiplication given by

$$
(v, z) \cdot\left(v^{\prime}, z^{\prime}\right)=\left(v+v^{\prime}, z+z^{\prime}+\frac{1}{2} \omega\left(v, v^{\prime}\right)\right) .
$$

The center of $H(V)$ is $Z=Z_{H(V)}=\left\{(0, z): z \in \mathbb{F}_{q}\right\}$. The symplectic group $\operatorname{Sp}(V)=\operatorname{Sp}(V, \omega)$ acts by automorphism on $H(V)$ through its tautological action on the $V$-coordinate.

1.2. The Heisenberg representation. One of the most important attributes of the group $H(V)$ is that it admits, principally, a unique irreducible representation - this is the $\mathrm{S}-\mathrm{vN}$ property. The precise statement goes as follows. Let $\psi: Z \rightarrow \mathbb{C}^{\times}$be a non-trivial character of the center. For example we can take $\psi(z)=\mathrm{e}^{\frac{2 \pi \mathrm{i}}{p} \operatorname{tr}(z)}$.

Theorem 1.1 (S-vN property). There exists a unique (up to isomorphism) irreducible unitary representation $(\pi, H, \mathcal{H})$ with the center acting by $\psi$, i.e., $\pi_{\mid Z}=\psi \cdot \operatorname{Id}_{\mathcal{H}}$.

The representation $\pi$ which appears in the above theorem will be called the Heisenberg representation.

1.3. The Weil representation. A direct consequence of Theorem 1.1 is the existence of a projective representation $\tilde{\rho}: \operatorname{Sp}(V) \rightarrow \operatorname{PGL}(\mathcal{H})$. The construction of $\tilde{\rho}$ out of the Heisenberg representation $\pi$ is due to Weil [19] and it goes as follows. Considering the Heisenberg representation $\pi$ and an element $g \in \operatorname{Sp}(V)$, one can define a new representation $\pi^{g}$ acting on the same Hilbert space via $\pi^{g}(h)=\pi(g(h))$. Clearly both $\pi$ and $\pi^{g}$ have the same central character $\psi$ hence by Theorem 1.1 they are isomorphic. Since the space $\operatorname{Hom}_{H(V)}\left(\pi, \pi^{g}\right)$ is one dimensional, choosing for every $g \in \operatorname{Sp}(V)$ a non-zero representative $\tilde{\rho}(g) \in \operatorname{Hom}_{H(V)}\left(\pi, \pi^{g}\right)$ gives the required projective 
representation. In more concrete terms, the projective representation $\tilde{\rho}$ is characterized by the formula

$$
\tilde{\rho}(g) \pi(h) \tilde{\rho}\left(g^{-1}\right)=\pi(g(h)),
$$

for every $g \in \operatorname{Sp}(V)$ and $h \in H(V)$.

It is known $[\mathbf{7}, \mathbf{1 3}]$ that

Theorem 1.2 (The Weil representation). There exists a linear representation $\rho: \operatorname{Sp}(V) \rightarrow \mathrm{GL}(\mathcal{H})$ lying over $\tilde{\rho}$.

In the next section we will show that the linear representation $\rho$ appears as a consequence of the existence of a canonical vector space $\mathcal{H}(V)$ associated with the symplectic vector space $V$.

\section{Canonical vector space}

2.1. Models of the Heisenberg representation. Although the representation $\pi$ is unique, it admits a multitude of different models (realizations), in fact this is one of its most interesting and powerful attributes. These models appear in families, in this work we will be interested in a particular family of such models which are associated with Lagrangian subspaces in $V$.

Let $\operatorname{Lag}(V)$ denote the set of Lagrangian subspaces in $V$. Let $\mathbb{C}(H(V), \psi)$ denote the subspace of functions $f \in \mathbb{C}(H(V))$ satisfying the equivariance property $f(z \cdot h)=\psi(z) f(h)$ for every $z \in Z$.

We associate with each Lagrangian subspace $L \in \operatorname{Lag}(V)$ a model $\left(\pi_{L}, H(V), \mathcal{H}_{L}\right)$ of the Heisenberg representation as follows: The vector space $\mathcal{H}_{L}$ consists of functions $f \in \mathbb{C}(H(V), \psi)$ satisfying $f(l \cdot h)=f(h)$ for every $l \in L$ and the Heisenberg action is given by right translation $\pi_{L}(h)[f]\left(h^{\prime}\right)=f\left(h^{\prime} \cdot h\right)$ for every $f \in \mathcal{H}_{L}$.

Definition 2.1. An oriented Lagrangian $L^{\circ}$ is a pair $L^{\circ}=\left(L, o_{L}\right)$, where $L \in \operatorname{Lag}(V)$ and $o_{L} \in \bigwedge^{\text {top }} L$ is a non-zero vector.

Let us denote by $\operatorname{OLag}(V)$ the set of oriented Lagrangian subspaces in $V$. Similarly, we associate with each oriented Lagrangian $L^{\circ} \in \operatorname{OLag}(V)$ a model $\left(\pi_{L^{\circ}}, H, \mathcal{H}_{L^{\circ}}\right)$ of the Heisenberg representation, taking $\mathcal{H}_{L^{\circ}}=\mathcal{H}_{L}$ and $\pi_{L^{\circ}}=\pi_{L}$. The collection of models $\left\{\mathcal{H}_{L^{\circ}}\right\}$ forms a vector bundle $\mathfrak{H}(V) \rightarrow$ $\operatorname{OLag}(V)$ with fibers $\mathfrak{H}_{L^{\circ}}=\mathcal{H}_{L^{\circ}}$. The vector bundle $\mathfrak{H}=\mathfrak{H}(V)$ is equipped with an additional structure of an action $\pi_{L^{\circ}}$ of $H(V)$ on each fiber. This suggests the following terminology:

Definition 2.2. Let $k \in \mathbb{N}$. An $H(V)^{k}$-vector bundle on $\operatorname{OLag}(V)$ is a vector bundle $\mathfrak{E} \rightarrow \operatorname{OLag}(V)$ equipped with a fiberwise action $\pi_{L^{\circ}}: H(V)^{k} \rightarrow$ $\operatorname{GL}\left(\mathfrak{E}_{L^{\circ}}\right)$, for every $L^{\circ} \in \operatorname{OLag}(V)$.

In addition, our $\mathfrak{H}$ is equipped with a natural $\mathrm{Sp}(V)$-equivariant structure, defined as follows: For every $g \in \operatorname{Sp}(V)$, let $g^{*} \mathfrak{H}$ be the $H(V)$-vector bundle 
with fibers $g^{*} \mathfrak{H}_{L^{\circ}}=\mathcal{H}_{g L^{\circ}}$ and the $g$-twisted Heisenberg action $\pi_{L^{\circ}}^{g}: H(V) \rightarrow$ GL $\left(\mathcal{H}_{g L^{\circ}}\right)$, given by $\pi_{L^{\circ}}^{g}(h)=\pi_{g L^{\circ}}(g(h))$. The equivariant structure is the isomorphisms of $H(V)$-vector bundles

$$
\theta_{g}: g^{*} \mathfrak{H} \longrightarrow \mathfrak{H},
$$

which on the level of fibers, sends $f \in \mathcal{H}_{g L^{\circ}}$ to $f \circ g \in \mathcal{H}_{L^{\circ}}$.

2.2. The strong S-vN property. By Theorem 1.1 , for every pair $\left(M^{\circ}\right.$, $\left.L^{\circ}\right) \in \operatorname{OLag}(V)^{2}$, the models $\mathcal{H}_{L^{\circ}}$ and $\mathcal{H}_{M^{\circ}}$ are isomorphic as representations of $H(V)$, moreover, since the Heisenberg representation is irreducible, the vector space $\operatorname{Hom}_{H}\left(\mathcal{H}_{L^{\circ}}, \mathcal{H}_{M}^{\circ}\right)$ of intertwining morphisms is one dimensional. Roughly, the strong S-vN property asserts the existence of a distinguished element $F_{M^{\circ}, L^{\circ}} \in \operatorname{Hom}_{H}\left(\mathcal{H}_{L^{\circ}}, \mathcal{H}_{M^{\circ}}\right)$, for every pair $\left(M^{\circ}, L^{\circ}\right) \in \mathrm{OLag}(V)^{2}$. The precise statement involves the following definition:

Definition 2.3. Let $\mathfrak{E} \rightarrow \operatorname{OLag}(V)$ be an $H(V)^{k}$-vector bundle. A trivialization of $\mathfrak{E}$ is a system of intertwining isomorphisms $\left\{E_{M^{\circ}, L^{\circ}} \in\right.$ $\left.\operatorname{Hom}_{H(V)^{k}}\left(\mathfrak{E}_{L^{\circ}}, \mathfrak{E}_{M^{\circ}}\right):\left(M^{\circ}, L^{\circ}\right) \in \operatorname{OLag}(V)^{2}\right\}$ satisfying the following multiplicativity condition:

$$
E_{N^{\circ}, M^{\circ} \circ} \circ E_{M^{\circ}, L^{\circ}}=E_{N^{\circ}, L^{\circ}},
$$

for every $N^{\circ}, M^{\circ}, L^{\circ} \in \mathrm{OLag}(V)$.

Remark 2.4. Intuitively, a trivialization of a $H(V)^{k}$-vector bundle $\mathfrak{E} \rightarrow$ $\operatorname{OLag}(V)$ can be thought of as a flat connection, compatible with the Heisenberg action and admitting a trivial monodromy.

Theorem 2.5 (The strong S-vN property). The $H(V)$-vector bundle $\mathfrak{H}$ admits a natural trivialization $\left\{T_{M^{\circ}, L^{\circ}}\right\}$.

The intertwining morphisms $T_{M^{\circ}, L^{\circ}}$ in the above theorem will be referred to as the canonical intertwining morphisms.

2.3. A linear algebra proof of the strong S-vN property. The proof of Theorem 2.5 proceeds in several steps. First, we note that the vector bundle $\mathfrak{H}$ admits a natural partial trivialization: let us denote by $U_{2} \subset \operatorname{OLag}(V)^{2}$ the subset consisting of pairs of oriented Lagrangians $\left(M^{\circ}, L^{\circ}\right) \in \operatorname{OLag}(V)^{2}$ which are in general position, that is $L+M=V$. For every $\left(M^{\circ}, L^{\circ}\right) \in U_{2}$, define the intertwining morphisms

$$
T_{M^{\circ}, L^{\circ}}=A_{M^{\circ}, L^{\circ}} \cdot F_{M^{\circ}, L^{\circ}}
$$

where $F_{M^{\circ}, L^{\circ}}: \mathcal{H}_{L^{\circ}} \rightarrow \mathcal{H}_{M^{\circ}}$ is the averaging morphism given by

$$
F_{M^{\circ}, L^{\circ}}[f](h)=\sum_{m \in M} f(m \cdot h),
$$


for every $f \in \mathcal{H}_{L^{\circ}}$ and $A_{M^{\circ}, L^{\circ}}$ is a normalization constant given by

$$
A_{M^{\circ}, L^{\circ}}=\left(G_{1} / q\right)^{n} \sigma\left((-1)^{\left(\begin{array}{c}
n \\
2
\end{array}\right)} \omega_{\wedge}\left(o_{L}, o_{M}\right)\right),
$$

where

- $\sigma$ is the unique quadratic character (also called the Legendre character) of the multiplicative group $G_{\mathrm{m}}=\mathbb{F}_{q}^{\times}$.

- $G_{1}$ is the one dimensional Gauss sum

$$
G_{1}=\sum_{z \in \mathbb{F}_{q}} \psi\left(\frac{1}{2} z^{2}\right) .
$$

- $\omega_{\wedge}: \bigwedge^{\text {top }} L \times \bigwedge^{\text {top }} M \rightarrow \mathbb{F}_{q}$ is the pairing induced by the symplectic form.

Let us denote by $U_{3} \subset \operatorname{OLag}(V)^{3}$ the subset consisting of triples of oriented Lagrangians $\left(N^{\circ}, M^{\circ}, L^{\circ}\right)$ which are in general position pairwisely.

Proposition 2.6. For every $\left(N^{\circ}, M^{\circ}, L^{\circ}\right) \in U_{3}$

$$
T_{N^{\circ}, L^{\circ}}=T_{N^{\circ}, M^{\circ}} \circ T_{M^{\circ}, L^{\circ}} .
$$

For a proof, see Appendix A.

Theorem 2.5, now, follows from

Proposition 2.7. The sub-system $\left\{T_{M^{\circ}, L^{\circ}}:\left(M^{\circ}, L^{\circ}\right) \in U_{2}\right\}$ extends in a unique manner to a trivialization of $\mathfrak{H}$.

For a proof, see Appendix A.

We will refer to $\left\{T_{M^{\circ}, L^{\circ}}\right\}$ as the system of canonical intertwining morphisms.

\subsection{Explicit formulas for the canonical intertwining morphisms.} The canonical intertwining morphism $T_{M^{\circ}, L^{\circ}}$ can be written in a closed form for a general pair $\left(M^{\circ}, L^{\circ}\right) \in \operatorname{OLag}(V)^{2}$. In order to do that we need to fix some additional terminology: Denote $I=M \cap L$ and let $n_{I}=\frac{\operatorname{dim}\left(I^{\perp} / I\right)}{2}$.

There are canonical decompositions

$$
\begin{gathered}
\bigwedge^{\mathrm{top}} M=\bigwedge^{\mathrm{top}} I \bigotimes \bigwedge_{\mathrm{top}}^{\mathrm{top}} M / I, \\
\bigwedge^{\mathrm{top}} I \bigotimes \bigwedge^{\mathrm{top}} L / I .
\end{gathered}
$$

In terms of these decompositions, the orientations on $M$ and $L$ can be written in the form $o_{M}=\iota_{M} \otimes o_{M / I}$ and $o_{L}=\iota_{L} \otimes o_{L / I}$, respectively. Let $F_{M^{\circ}, L^{\circ}}: \mathcal{H}_{L^{\circ}} \rightarrow \mathcal{H}_{M^{\circ}}$ denote the averaging morphism

$$
F_{M^{\circ}, L^{\circ}}[f](h)=\sum_{\bar{m} \in M / I} f(m \cdot h),
$$


for every $f \in \mathcal{H}_{L^{\circ}}$ where in the above summation, $m \in M$ is any element lying over $\bar{m} \in M / I$. Define the normalization constant

$$
A_{M^{\circ}, L^{\circ}}=\left(G_{1} / q\right)^{n_{I}} \sigma\left((-1)^{\left(\begin{array}{c}
n_{I} \\
2
\end{array}\right)} \frac{\iota_{L}}{\iota_{M}} \cdot \omega_{\wedge}\left(o_{L / I}, o_{M / I}\right)\right),
$$

where $\omega_{\wedge}: \bigwedge^{\text {top }} L / I \times \bigwedge^{\text {top }} M / I \rightarrow \mathbb{F}_{q}$ is the pairing induced by the symplectic form $\omega$.

Proposition 2.8. For every $\left(M^{\circ}, L^{\circ}\right) \in \operatorname{OLag}(V)^{2}$

$$
T_{M^{\circ}, L^{\circ}}=A_{M^{\circ}, L^{\circ}} \cdot F_{M^{\circ}, L^{\circ}} .
$$

For a proof, see Appendix A.

2.5. Kernel presentation of an intertwining morphism. An explicit way to present an intertwining morphism is via a kernel function. Fix a pair $\left(M^{\circ}, L^{\circ}\right) \in \operatorname{OLag}(V)^{2}$ of oriented Lagrangians and let $\mathbb{C}(M \backslash H(V) / L, \psi) \subset$ $\mathbb{C}(H(V), \psi)$ be the subspace of functions $K \in \mathbb{C}(H(V), \psi)$, satisfying the equivariance property $K(m \cdot h \cdot l)=K(h)$, for every $m \in M$ and $l \in L$. Given a function $K \in \mathbb{C}(M \backslash H(V) / L, \psi)$, we can associate to it the intertwining morphism $I[K] \in \operatorname{Hom}_{H(V)}\left(\mathcal{H}_{L^{\circ}}, \mathcal{H}_{M^{\circ}}\right)$ defined by

$$
I[K](f)=K * f=m_{!}\left(K \nabla_{Z \cdot L} f\right),
$$

for every $f \in \mathcal{H}_{L^{\circ}}$, where $K \otimes_{Z \cdot L} f$ is the descent of the function $K \otimes f \in$ $\mathbb{C}(H(V) \times H(V))$ to $H(V) \times{ }_{Z \cdot L} H(V)$ - the quotient of $H(V) \times H(V)$ by the action $x \cdot\left(h_{1}, h_{2}\right)=\left(h_{1} x, x^{-1} h_{2}\right)$ for $x \in Z \cdot L$ - and $m_{!}$denotes the operation of summation along the fibers of the multiplication mapping $m$ : $H(V) \times H(V) \rightarrow H(V)$. We call the function $K$ an intertwining kernel. The procedure that we just described defines an isomorphism of vector spaces

$$
I: \mathbb{C}(M \backslash H(V) / L, \psi) \longrightarrow \operatorname{Hom}_{H(V)}\left(\mathcal{H}_{L^{\circ}}, \mathcal{H}_{M^{\circ}}\right) .
$$

Fix a triple $\left(N^{\circ}, M^{\circ}, L^{\circ}\right) \in \operatorname{OLag}(V)^{3}$. Given kernels $K_{1} \in \mathbb{C}(N \backslash H(V) /$ $M, \psi)$ and $K_{2} \in \mathbb{C}(M \backslash H(V) / L, \psi)$, their convolution $K_{1} * K_{2}=m_{\text {! }}$ $\left(K_{1} \otimes_{Z \cdot M} K_{2}\right)$ lies in $\mathbb{C}(N \backslash H(V) / L, \psi)$. Moreover, the transform $I$ sends convolution of kernels to composition of operators

$$
I\left[K_{1} * K_{2}\right]=I\left[K_{1}\right] \circ I\left[K_{2}\right] .
$$

2.5.1. Canonical system of intertwining kernels. Below, we formulate a version of Theorem 2.5 in the setting of kernels.

Definition 2.9. A system $\left\{K_{M^{\circ}, L^{\circ}} \in \mathbb{C}(M \backslash H(V) / L, \psi):\left(M^{\circ}, L^{\circ}\right) \in\right.$ $\left.\operatorname{OLag}(V)^{2}\right\}$ of kernels is called multiplicative if for every triple $\left(N^{\circ}, M^{\circ}, L^{\circ}\right)$ $\in \operatorname{OLag}(V)^{3}$ the following equation holds:

$$
K_{N^{\circ}, L^{\circ}}=K_{N^{\circ}, M^{\circ}} * K_{M^{\circ}, L^{\circ}}
$$


A multiplicative system of kernels $\left\{K_{M^{\circ}, L^{\circ}}\right\}$ can be equivalently thought of as a single function $K \in \mathbb{C}\left(\operatorname{OLag}(V)^{2} \times H(V)\right)$ defined by $K\left(M^{\circ}, L^{\circ},-\right)=$ $K_{M^{\circ}, L^{\circ}}(-)$ satisfying the following multiplicativity relation on $\operatorname{OLag}(V)^{3} \times$ $H(V)$

$$
p_{12}^{*} K * p_{23}^{*} K=p_{13}^{*} K,
$$

where $p_{i j}\left(\left(L_{1}^{\circ}, L_{2}^{\circ}, L_{3}^{\circ}\right), h\right)=\left(\left(L_{i}^{\circ}, L_{j}^{\circ}\right), h\right)$ are the projections on the $i, j$ copies of $\operatorname{OLag}(V)$ and the left-hand side of (2.4) means fiberwise convolution, namely $p_{12}^{*} K * p_{23}^{*} K\left(L_{1}^{\circ}, L_{2}^{\circ}, L_{3}^{\circ},-\right)=K\left(L_{1}^{\circ}, L_{2}^{\circ},-\right) * K\left(L_{2}^{\circ}, L_{3}^{\circ},-\right)$.

For every $\left(M^{\circ}, L^{\circ}\right) \in \mathrm{OLag}(V)^{2}$, there exists a unique kernel $K_{M^{\circ}, L^{\circ}}$ such that $T_{M^{\circ}, L^{\circ}}=I\left[K_{M^{\circ}, L^{\circ}}\right]$. We will refer to $\left\{K_{M^{\circ}, L^{\circ}}\right\}$ as the system of canonical intertwining kernels. We will denote the corresponding function on $\operatorname{OLag}(V)^{2} \times H(V)$ by $K$.

Proposition 2.10. The function $K \in \mathbb{C}\left(\mathrm{OLag}(V)^{2} \times H(V)\right)$ satisfies

$$
p_{12}^{*} K * p_{23}^{*} K=p_{13}^{*} K \text {. }
$$

In case $\left(M^{\circ}, L^{\circ}\right) \in U_{2}$, the kernel $K_{M^{\circ}, L^{\circ}}$ is given by the following explicit formula

$$
K_{M^{\circ}, L^{\circ}}=A_{M^{\circ}, L^{\circ}} \cdot \tilde{K}_{M^{\circ}, L^{\circ}},
$$

where $\tilde{K}_{M^{\circ}, L^{\circ}}=\left(\tau^{-1}\right)^{*} \psi$ where $\tau=\tau_{M^{\circ}, L^{\circ}}$ is the isomorphism given by the composition $Z \hookrightarrow H \rightarrow M \backslash H(V) / L$. The system $\left\{K_{M^{\circ}, L^{\circ}}:\left(M^{\circ}, L^{\circ}\right) \in U_{2}\right\}$ yields a well-defined function $K_{U_{2}} \in \mathbb{C}\left(U_{2} \times H(V)\right)$. In Section 3 , we will give an algebra geometric interpretation to the description of the kernels $K_{M^{\circ}, L^{\circ}}$ when $\left(M^{\circ}, L^{\circ}\right) \notin U_{2}$.

2.6. The canonical vector space. Let us denote by Symp the category whose objects are finite-dimensional symplectic vector spaces over the finite field $\mathbb{F}_{q}$ and morphisms are linear isomorphisms of symplectic vector spaces. In addition, let us denote by Vect the category of finite-dimensional complex vector spaces. Using Theorem 2.5, we can associate, in a functorial manner, a vector space $\mathcal{H}(V)$ to a symplectic vector space $V \in$ Symp as follows: Define $\mathcal{H}(V)$ to be the space of "horizontal sections" of the trivialized vector bundle $\mathfrak{H}(V)$

$$
\mathcal{H}(V)=\Gamma_{\text {hor }}(\operatorname{OLag}(V), \mathfrak{H}(V)),
$$

where $\Gamma_{\text {hor }}(\operatorname{OLag}(V), \mathfrak{H}(V)) \subset \Gamma(\operatorname{OLag}(V), \mathfrak{H}(V))$ is the sub-space consisting of sections $\left(v_{L^{\circ}} \in \mathcal{H}_{L^{\circ}}: L^{\circ} \in \mathrm{OLag}(V)\right)$ satisfying $T_{M^{\circ}, L^{\circ}}\left(v_{L^{\circ}}\right)=v_{M^{\circ}}$ for every $\left(M^{\circ}, L^{\circ}\right) \in \mathrm{OLag}(V)^{2}$. The vector space $\mathcal{H}(V)$ will be referred to as the canonical vector space associated with $V$.

Notation 2.11. The definition of the vector space $\mathcal{H}(V)$ depends on a choice of a central character $\psi$. Sometime, we will use the notation $\mathcal{H}(V, \psi)$ to emphasize this point. 
Proposition 2.12 (Functoriality). The rule $V \mapsto \mathcal{H}(V)$ establishes a contravariant (quantization) functor

$$
\mathcal{H}: \text { Symp } \longrightarrow \text { Vect. }
$$

For a proof, see Appendix A.

Considering a fixed symplectic vector space $V$, we obtain as a consequence a representation $\left(\rho_{V}, \operatorname{Sp}(V), \mathcal{H}(V)\right)$, with $\rho_{V}(g)=\mathcal{H}\left(g^{-1}\right)$, for every $g \in$ $\operatorname{Sp}(V)$. The representation $\rho_{V}$ is isomorphic to the Weil representation and will be referred to as the canonical model of the Weil representation.

Remark 2.13. The canonical model $\rho_{V}$ can be viewed from another perspective: We begin with the total vector space $\Gamma(V)=\Gamma(\operatorname{OLag}(V), \mathfrak{H}(V))$ and make the following two observations. First observation, is that the symplectic group $\operatorname{Sp}(V)$ acts naturally on $\Gamma(V)$, the action is of a geometric nature - induced from the diagonal action on $\operatorname{OLag}(V) \times H(V)$. Second observation, is that the system $\left\{T_{M^{\circ}, L^{\circ}}\right\}$ defines an $\operatorname{Sp}(V)$-invariant idempotent, which should be thought of as a total Fourier transform, $T: \Gamma(V) \rightarrow \Gamma(V)$ given by

$$
T\left(v_{L^{\circ}}\right)=\frac{1}{\#(\operatorname{OLag}(V))} \bigoplus_{M^{\circ} \in \operatorname{OLag}(V)} T_{M^{\circ}, L^{\circ}}\left(v_{L^{\circ}}\right),
$$

for every $L^{\circ} \in \mathrm{OLag}(V)$ and $v_{L^{\circ}} \in \mathcal{H}_{L^{\circ}}$. The situation is summarized in the following diagram:

$$
\operatorname{Sp}(V) \circlearrowright \Gamma(V) \circlearrowleft T .
$$

The canonical model is given by the image of $T$, that is, $\mathcal{H}(V)=T(\Gamma(V))$. The nice thing about this point of view is that it shows a clear distinction between operators associated with action of the symplectic group and operators associated with intertwining morphisms. Finally, we remark that we can, also, consider the $\operatorname{Sp}(V)$-invariant idempotent $T^{\perp}=\mathrm{Id}-T$ and the associated representation $\left(\rho_{V}^{\perp}, \operatorname{Sp}(V), \mathcal{H}(V)^{\perp}\right)$, with $\mathcal{H}(V)^{\perp}=T^{\perp}(\Gamma(V))$. The meaning of this representation is unclear.

\subsection{Properties of the quantization functor}

2.7.1. Compatibility with Cartesian products. The category Symp admits a monoidal structure given by Cartesian product of symplectic vector spaces. The category Vect admits the standard monoidal structure given by tensor product. The functor $\mathcal{H}$ is a monoidal functor with respect to these monoidal structures.

Proposition 2.14. For every $V_{1}, V_{2} \in$ Symp, there exists a natural isomorphism

$$
\alpha_{V_{1} \times V_{2}}: \mathcal{H}\left(V_{1} \times V_{2}\right) \stackrel{\simeq}{\rightarrow} \mathcal{H}\left(V_{1}\right) \otimes \mathcal{H}\left(V_{2}\right)
$$


For a proof, see Appendix A.

As a result, we obtain the following compatibility condition between the canonical models of the Weil representation

$$
\alpha_{V_{1} \times V_{2}}:\left(\rho_{V_{1} \times V_{2}}\right)_{\mid \operatorname{Sp}\left(V_{1}\right) \times \operatorname{Sp}\left(V_{2}\right)} \stackrel{\simeq}{\longrightarrow} \rho_{V_{1}} \otimes \rho_{V_{2}} .
$$

Remark 2.15. Condition (2.6) has an interesting consequence in case the ground field is $\mathbb{F}_{3}[\mathbf{5}]$. In this case, the group $\operatorname{Sp}(V)$ is not perfect when $\operatorname{dim} V=2$, therefore, in this particular situation, the Weil representation is not uniquely defined. Nevertheless, since the group $\operatorname{Sp}(V)$ becomes perfect when $\operatorname{dim} V>2$, the canonical model gives a natural choice for the Weil representation in the "singular" dimension, $\operatorname{dim} V=2$.

2.7.2. Compatibility with symplectic duality. Let $V=(V, \omega) \in$ Symp and let us denote by $\bar{V}=(V,-\omega)$ the symplectic dual of $V$.

Proposition 2.16. There exists a natural non-degenerate pairing

$$
\langle\cdot, \cdot\rangle_{V}: \mathcal{H}(\bar{V}) \times \mathcal{H}(V) \longrightarrow \mathbb{C},
$$

where $V \in$ Symp.

For a proof, see Appendix A.

2.7.3. Compatibility with symplectic reduction. Let $V \in$ Symp. Let $I$ be an isotropic subspace in $V$ considered as an abelian subgroup in $H(V)$. The fiberwise action of $H(V)$ on the vector bundle $\mathfrak{H}$ induces an action of $H(V)$ on $\mathcal{H}(V)$, using this action we can associate to $I$ the subspace $\mathcal{H}(V)^{I}$ of $I$-invariant vectors. In addition, we can form the symplectic reduction ${ }^{2}$ $I^{\perp} / I$ and consider the canonical vector space $\mathcal{H}\left(I^{\perp} / I\right)$. Roughly, we claim that the vector spaces $\mathcal{H}\left(I^{\perp} / I\right)$ and $\mathcal{H}(V)^{I}$ are naturally isomorphic. The precise statement involves the following definition

Definition 2.17. An oriented isotropic subspace in $V$ is a pair $I^{\circ}=\left(I, o_{I}\right)$, where $I \subset V$ is an isotropic subspace and $o_{I} \in \bigwedge^{\text {top }} I$ is a non-trivial vector.

Proposition 2.18. There exists a natural isomorphism

$$
\alpha_{\left(I^{\circ}, V\right)}: \mathcal{H}(V)^{I} \stackrel{\simeq}{\rightarrow} \mathcal{H}\left(I^{\perp} / I\right)
$$

where $V \in$ Symp and $I^{\circ}$ an oriented isotropic subspace in $V$. The naturality condition is

$$
\mathcal{H}\left(f_{I}\right) \circ \alpha_{\left(J^{\circ}, U\right)}=\alpha_{\left(I^{\circ}, V\right)} \circ \mathcal{H}(f),
$$

for every $f \in \operatorname{Mor}_{\text {Symp }}(V, U)$ such that $f\left(I^{\circ}\right)=J^{\circ}$ and $f_{I} \in \operatorname{Mor}_{\text {Symp }}\left(I^{\perp} / I\right.$, $\left.J^{\perp} / J\right)$ is the induced morphism.

\footnotetext{
${ }^{2}$ Note that since $I$ is isotropic, $I \subset I^{\perp}$ and $I^{\perp} / I$ is equipped with a natural symplectic structure.
} 
For a proof, see Appendix A.

As a result, we obtain the following compatibility condition between the canonical models of the Weil representation: Fix $V \in$ Symp and let $I^{\circ}$ be an enhanced isotropic subspace in $V$. Let $P \subset \mathrm{Sp}(V)$ be the subgroup of elements $g \in \operatorname{Sp}(V)$ such that $g\left(I^{\circ}\right)=I^{\circ}$. The isomorphism $\alpha_{\left(I^{\circ}, V\right)}$ establishes the following isomorphism:

$$
\alpha_{\left(I^{\circ}, V\right)}:\left(\rho_{V}\right)_{\mid P} \stackrel{\simeq}{\longrightarrow} \rho_{I^{\perp} / I^{\circ}} \circ \pi
$$

where $\pi: P \rightarrow \operatorname{Sp}\left(I^{\perp} / I\right)$ is the canonical homomorphism.

\section{Geometric canonical intertwining kernels}

In this section, we construct a geometric counterpart to the set-theoretic system of canonical intertwining kernels. In particular, we obtain an algebrageometric interpretation for the kernels $K_{M^{\circ}, L^{\circ}}$ when $\left(M^{\circ}, L^{\circ}\right) \notin U_{2}$, and an alternative proof for the strong $\mathrm{S}-\mathrm{vN}$ theorem.

3.1. Preliminaries from algebraic geometry. We denote by $k$ an algebraic closure of the field $\mathbb{F}_{q}$. Next, we have to use some space to recall notions and notations from algebraic geometry and the theory of $\ell$-adic sheaves.

3.1.1. Varieties. In the sequel, we are going to translate back and forth between algebraic varieties defined over the finite field $\mathbb{F}_{q}$ and their corresponding sets of rational points. In order to prevent confusion between the two, we use bold-face letters to denote a variety $\mathbf{X}$ and normal letters $X$ to denote its corresponding set of rational points $X=\mathbf{X}\left(\mathbb{F}_{q}\right)$. For us, a variety $\mathbf{X}$ over the finite field is a quasi-projective algebraic variety, such that the defining equations are given by homogeneous polynomials with coefficients in the finite field $\mathbb{F}_{q}$. In this situation, there exists a (geometric) Frobenius endomorphism Fr : $\mathbf{X} \rightarrow \mathbf{X}$, which is a morphism of algebraic varieties. We denote by $X$ the set of points fixed by Fr, i.e.,

$$
X=\mathbf{X}\left(\mathbb{F}_{q}\right)=\mathbf{X}^{\mathrm{Fr}}=\{x \in \mathbf{X}: \operatorname{Fr}(x)=x\} .
$$

The category of algebraic varieties over $\mathbb{F}_{q}$ will be denoted by $\operatorname{Var}_{\mathbb{F}_{q}}$.

3.1.2. Sheaves. Let $D^{\mathrm{b}}(\mathbf{X})$ denote the bounded derived category of constructible $\ell$-adic sheaves on $\mathbf{X}[\mathbf{2}]$. We denote by $\operatorname{Perv}(\mathbf{X})$ the Abelian category of perverse sheaves on the variety $\mathbf{X}$, that is the heart with respect to the autodual perverse t-structure in $\mathrm{D}^{\mathrm{b}}(\mathbf{X})$. An object $\mathcal{F} \in \mathrm{D}^{\mathrm{b}}(\mathbf{X})$ is called $N$-perverse if $\mathcal{F}[N] \in \operatorname{Perv}(\mathbf{X})$. Finally, we recall the notion of a Weil structure (Frobenius structure) [4]. A Weil structure associated to an object $\mathcal{F} \in \mathrm{D}^{\mathrm{b}}(\mathbf{X})$ is an isomorphism

$$
\theta: \operatorname{Fr}^{*} \mathcal{F} \stackrel{\sim}{\longrightarrow} \mathcal{F} .
$$


A pair $(\mathcal{F}, \theta)$ is called a Weil object. By an abuse of notation we often denote $\theta$ also by Fr. We choose once an identification $\overline{\mathbb{Q}}_{\ell} \simeq \mathbb{C}$, hence all sheaves are considered over the complex numbers.

Remark 3.1. All the results in this section make perfect sense over the field $\overline{\mathbb{Q}}_{\ell}$, in this respect the identification of $\overline{\mathbb{Q}}_{\ell}$ with $\mathbb{C}$ is redundant. The reason it is specified is in order to relate our results with the standard constructions.

Given a Weil object $\left(\mathcal{F}, \operatorname{Fr}^{*} \mathcal{F} \simeq \mathcal{F}\right)$ one can associate to it a function $f^{\mathcal{F}}: X \rightarrow \mathbb{C}$ to $\mathcal{F}$ as follows:

$$
f^{\mathcal{F}}(x)=\sum_{i}(-1)^{i} \operatorname{Tr}\left(\operatorname{Fr}_{\mid H^{i}\left(\mathcal{F}_{x}\right)}\right) .
$$

This procedure is called Grothendieck's sheaf-to-function correspondence [10]. Another common notation for the function $f^{\mathcal{F}}$ is $\chi_{\mathrm{Fr}}(\mathcal{F})$, which is called the Euler characteristic of the sheaf $\mathcal{F}$.

3.2. Geometrization. We shall now start the geometrization procedure.

3.2.1. Replacing sets by varieties. The first step we take is to replace all sets involved by their geometric counterparts, i.e., algebraic varieties. The symplectic space $(V, \omega)$ is naturally identified as the set $V=\mathbf{V}\left(\mathbb{F}_{q}\right)$, where $\mathbf{V}$ is a $2 n$-dimensional symplectic vector space in $\operatorname{Var}_{\mathbb{F}_{q}}$. The Heisenberg group $H$ is naturally identified as the set $H=\mathbf{H}\left(\mathbb{F}_{q}\right)$, where $\mathbf{H}=\mathbf{V} \times \mathbb{G}_{\mathrm{a}}$ is the corresponding group variety. Finally, $\operatorname{OLag}(V)=\mathbf{O L a g}(\mathbf{V})\left(\mathbb{F}_{q}\right)$, where $\operatorname{OLag}(\mathbf{V})$ is the variety of oriented Lagrangians in $\mathbf{V}$.

3.2.2. Replacing functions by sheaves. The second step is to replace functions by their sheaf-theoretic counterparts $[\mathbf{6}]$. The additive character $\psi: \mathbb{F}_{q} \longrightarrow \mathbb{C}^{\times}$is associated via the sheaf-to-function correspondence to the Artin-Schreier sheaf $\mathcal{L}_{\psi}$ on the variety $\mathbb{G}_{\mathrm{a}}$, i.e., we have $f^{\mathcal{L}_{\psi}}=\psi$. The Legendre character $\sigma$ on $\mathbb{F}_{q}^{\times} \simeq \mathbb{G}_{\mathrm{m}}\left(\mathbb{F}_{q}\right)$ is associated to the Kummer sheaf $\mathcal{L}_{\sigma}$ on the variety $\mathbb{G}_{\mathrm{m}}$. The one-dimensional Gauss sum $G_{1}$ is associated with the Weil object

$$
\mathcal{G}_{1}=\int_{\mathbb{G}_{\mathrm{a}}} \mathcal{L}_{\psi\left((1 / 2) z^{2}\right)} \in \mathrm{D}^{\mathrm{b}}(\mathbf{p t})
$$

where, for the rest of this paper, $\int=\int_{\text {! }}$ denotes integration with compact support [2]. Grothendieck's Lefschetz trace formula [10] implies that, indeed, $f^{\mathcal{G}_{1}}=G_{1}$. In fact, there exists a quasi-isomorphism $\mathcal{G}_{1} \stackrel{q . i}{\longrightarrow} H^{1}\left(\mathcal{G}_{1}\right)[-1]$ and $\operatorname{dim} H^{1}\left(\mathcal{G}_{1}\right)=1$, hence, $\mathcal{G}_{1}$ can be thought of as a one-dimensional vector space, equipped with a Frobenius operator, sitting at cohomological degree 1.

3.3. Canonical system of geometric intertwining kernels. Let $\mathbf{U}_{2} \subset$ $\operatorname{OLag}(\mathbf{V})^{2}$ be the open subvariety consisting of pairs $\left(M^{\circ}, L^{\circ}\right) \in \mathbf{O L a g}(\mathbf{V})^{2}$ 
which are in general position. We define a sheaf "of kernels" $\mathcal{K}_{\mathbf{U}_{2}}$ on the variety $\mathbf{U}_{2} \times \mathbf{H}(\mathbf{V})$ as follows:

$$
\mathcal{K}_{\mathbf{U}_{2}}=\mathcal{A} \otimes \tilde{\mathcal{K}}_{\mathbf{U}_{2}}
$$

where

- $\tilde{\mathcal{K}}_{\mathbf{U}_{2}}$ is the sheaf of non-normalized kernels given by

$$
\tilde{\mathcal{K}}_{\mathbf{U}_{2}}\left(M^{\circ}, L^{\circ}\right)=\left(\tau^{-1}\right)^{*} \mathcal{L}_{\psi},
$$

where $\tau=\tau_{M^{\circ}, L^{\circ}}$ is the isomorphism given by the composition

$$
\mathbf{Z} \hookrightarrow \mathbf{H} \rightarrow \mathbf{M} \backslash \mathbf{H} / \mathbf{L} .
$$

- $\mathcal{A}$ is the "Normalization coefficient" sheaf given by

$$
\mathcal{A}\left(M^{\circ}, L^{\circ}\right)=\mathcal{G}_{1}^{\otimes n} \otimes \mathcal{L}_{\sigma}\left((-1)^{\left(\begin{array}{c}
n \\
2
\end{array}\right)} \omega_{\wedge}\left(o_{L}, o_{M}\right)\right)[2 n](n) .
$$

Let $n_{k}=\operatorname{dim}\left(\mathbf{O L a g}(\mathbf{V})^{k}\right)+n+1$ for $k \in \mathbb{N}$. By construction, the sheaf $\mathcal{K}_{\mathbf{U}_{2}}$ is a local system (lissé) of rank 1 , normalized to sit in cohomological degree $-n_{2}$ and consequently of pure weight $w\left(\mathcal{K}_{\mathbf{U}_{2}}\right)=0$.

Proposition 3.2. The local system $\mathcal{K}_{\mathbf{U}_{2}}$ can be extended in a unique manner to a geometrically irreducible $\left[n_{2}\right]$-perverse Weil sheaf $\mathcal{K}$ on $\mathbf{O L a g}(\mathbf{V})^{2} \times$ $\mathbf{H}(\mathbf{V})$ of pure weight $w(\mathcal{K})=0$. Moreover, there exists an isomorphism

$$
p_{13}^{*} \mathcal{K} \simeq p_{12}^{*} \mathcal{K} * p_{23}^{*} \mathcal{K} .
$$

The proof of Proposition 3.2 proceeds in several steps. The construction of $\mathcal{K}$ uses the functor of middle extension, namely, take

$$
\mathcal{K}=j_{! *} \mathcal{K}_{\mathbf{U}_{2}}
$$

where $j: \mathbf{U}_{2} \times \mathbf{H}(\mathbf{V}) \hookrightarrow \mathbf{O L a g}(\mathbf{V})^{2} \times \mathbf{H}(\mathbf{V})$ is the open imbedding and $j_{\text {!* }}$ is the functor of middle extension $[\mathbf{2}]$. It follows directly from the construction that the sheaf $\mathcal{K}$ is irreducible $\left[n_{2}\right]$-perverse of pure weight 0 .

Remark 3.3. Proposition 3.2 establishes an alternative proof of Proposition 2.10 as follows: let $K=f^{\mathcal{K}}$. The multiplicativity condition for the sheaf $\mathcal{K}$ implies that $K$ is multiplicative. Moreover, since $K_{U_{2}}=f^{\mathcal{K}_{\mathbf{U}_{2}}}$, it implies that $K$ extends the function $K_{U_{2}}$. In addition, the geometric construction yields a description of the kernels $K_{M^{\circ}, L^{\circ}}$ when $\left(M^{\circ}, L^{\circ}\right) \notin U_{2}$ in terms of the middle extension of $\mathcal{K}_{\mathbf{U}_{2}}$.

3.4. Proof of the multiplicativity property. Denote by $\mathbf{U}_{3} \subset$ OLag $(\mathbf{V})^{3}$ the open subvariety consisting of triples $\left(N^{\circ}, M^{\circ}, L^{\circ}\right)$ which are in general position pairwisely.

Lemma 3.4. There exists an isomorphism on $\mathbf{U}_{3} \times \mathbf{H}(\mathbf{V})$

$$
p_{13}^{*} \mathcal{K} \simeq p_{12}^{*} \mathcal{K} * p_{23}^{*} \mathcal{K} \text {. }
$$


For a proof, see Appendix A.

Let $\mathbf{V}_{3} \subset \mathbf{O L a g}(\mathbf{V})^{3}$ denote the open subvariety consisting of triples $\left(N^{\circ}, M^{\circ}, L^{\circ}\right)$ such that $\left(N^{\circ}, M^{\circ}\right),\left(M^{\circ}, L^{\circ}\right) \in \mathbf{U}_{2}$.

Lemma 3.5. There exists an isomorphism on $\boldsymbol{V}_{3} \times \boldsymbol{H}(\boldsymbol{V})$

$$
p_{13}^{*} \mathcal{K} \simeq p_{12}^{*} \mathcal{K} * p_{23}^{*} \mathcal{K} .
$$

For a proof, see Appendix A.

Using Lemma 3.4 we conclude that the sheaves $p_{13}^{*} \mathcal{K}$ and $p_{12}^{*} \mathcal{K} * p_{23}^{*} \mathcal{K}$ are isomorphic on the open subvariety $\mathbf{U}_{3} \times \mathbf{H}(\mathbf{V})$. The sheaf $p_{13}^{*} \mathcal{K}$ is irreducible $\left[n_{3}\right]$-perverse as a pull-back by a smooth, surjective with connected fibers morphism, of an irreducible $\left[n_{2}\right]$-perverse sheaf on $\operatorname{OLag}(\mathbf{V})^{2} \times$ $\mathbf{H}(\mathbf{V})$, hence, it is enough to show that the sheaf $p_{12}^{*} \mathcal{K} * p_{23}^{*} \mathcal{K}$ is irreducible $\left[n_{3}\right]$-perverse.

Let us denote by $\mathbf{V}_{4} \subset \mathbf{O L a g}(\mathbf{V})^{4}$ the open subvariety consisting of quadruples $\left(N^{\circ}, S^{\circ}, M^{\circ}, L^{\circ}\right) \in \mathbf{O L a g}(\mathbf{V})^{4}$ such that $\left(N^{\circ}, S^{\circ}\right),\left(S^{\circ}, M^{\circ}\right) \in$ $\mathbf{U}_{2}$. The projection $p_{134}: \mathbf{V}_{4} \times \mathbf{H}(\mathbf{V}) \rightarrow \operatorname{OLag}(\mathbf{V})^{3} \times \mathbf{H}(\mathbf{V})$ is smooth, surjective and admits connected fibers, therefore, it is enough to show that the pull-back $p_{134}^{*}\left(p_{12}^{*} \mathcal{K} * p_{23}^{*} \mathcal{K}\right)$ is irreducible $\left[n_{4}\right]$-perverse. Using Lemma 3.5 and also invoking some direct diagram chasing we obtain

$$
p_{134}^{*}\left(p_{12}^{*} \mathcal{K} * p_{23}^{*} \mathcal{K}\right) \simeq p_{12}^{*} \mathcal{K} * p_{23}^{*} \mathcal{K} * p_{34}^{*} \mathcal{K} .
$$

The right-hand side of the above formula is principally a subsequent application of properly normalized, Fourier transforms (see formula (A.6) below) on $p_{34}^{*} \mathcal{K}$, hence by the Katz-Laumon theorem [16] it is irreducible $\left[n_{4}\right]$ perverse.

Let us summarize. We showed that both sheaves $p_{13}^{*} \mathcal{K}$ and $p_{12}^{*} \mathcal{K} * p_{23}^{*} \mathcal{K}$ are irreducible $\left[n_{3}\right]$-perverse and are isomorphic on an open subvariety. This implies that they must be isomorphic.

This concludes the proof of the multiplicativity property.

\section{Appendix A. Proofs of statements}

A.1. Proof of Proposition 2.6. For every $\left(N^{\circ}, M^{\circ}, L^{\circ}\right) \in U_{3}$ the intertwining morphisms $F_{N^{\circ}, M^{\circ}} \circ F_{M^{\circ}, L^{\circ}}, F_{N^{\circ}, L^{\circ}} \in \operatorname{Hom}_{H(V)}\left(\mathcal{H}_{L^{\circ}}, \mathcal{H}_{N^{\circ}}\right)$ are proportional, namely

$$
F_{N^{\circ}, M^{\circ} \circ} \circ F_{M^{\circ}, L^{\circ}}=C\left(N^{\circ}, M^{\circ}, L^{\circ}\right) \cdot F_{N^{\circ}, L^{\circ}},
$$

for some $C\left(N^{\circ}, M^{\circ}, L^{\circ}\right) \in \mathbb{C}$. This follows from the fact that $\mathcal{H}_{L^{\circ}}$ and $\mathcal{H}_{N^{\circ}}$ are irreducible and isomorphic as representations of the Heisenberg group $H(V)$.

The proof will proceed in two steps.

Step 1. We calculate the proportionality coefficient $C\left(N^{\circ}, M^{\circ}, L^{\circ}\right)$. 
Let $\delta_{L} \in \mathcal{H}_{L^{\circ}}$ denote the unique function supported on $L \cdot Z$ normalized such that $\delta_{L}(0)=1$. On the one hand, it is easy to verify that $F_{N^{\circ}, L^{\circ}}\left(\delta_{L}\right)(0)=1$ therefore

$$
C\left(N^{\circ}, M^{\circ}, L^{\circ}\right)=F_{N^{\circ}, M^{\circ} \circ} F_{M^{\circ}, L^{\circ}}\left(\delta_{L}\right)(0) .
$$

On the other hand, explicit calculation reveals that

$$
F_{N^{\circ}, M^{\circ} \circ} \circ F_{M^{\circ}, L^{\circ}}\left(\delta_{L}\right)(0)=\sum_{m \in M} \psi\left(\frac{1}{2} \omega\left(r^{L}(m), m\right),\right.
$$

where $r^{L}: M \rightarrow N$ is the linear map defined by the condition $r^{L}(m)-m \in L$ for every $m \in M$. Furthermore, an easy diagonalization argument implies that

$$
\sum_{m \in M} \psi\left(\frac{1}{2} \omega\left(r^{L}(m), m\right)\right)=G_{1}^{n} \cdot \sigma\left(d\left[\omega\left(r^{L}(-),-\right)\right]\right),
$$

where $d\left[\omega\left(r^{L}(-),-\right)\right] \in \mathbb{F}_{q}^{\times} / \mathbb{F}_{q}^{\times 2}$ denotes the discriminant of the symmetric form $\omega\left(r^{L}(-),-\right)$. The map $r^{L}: M \rightarrow N$ induces a map $r_{\wedge}^{L}: \bigwedge^{\text {top }} M \rightarrow$ $\bigwedge^{\text {top }} N$.

Lemma A.1. We have

$$
d\left[\omega\left(r^{L}(-),-\right)\right]=(-1)^{\left(\begin{array}{l}
n \\
2
\end{array}\right)} \omega_{\wedge}\left(r_{\wedge}^{L}\left(o_{M}\right), o_{M}\right) .
$$

Summarizing, we get that

$$
C\left(N^{\circ}, M^{\circ}, L^{\circ}\right)=G_{1}^{n} \cdot \sigma\left((-1)^{\left(\begin{array}{c}
n \\
2
\end{array}\right)} \omega_{\wedge}\left(r_{\wedge}^{L}\left(o_{M}\right), o_{M}\right)\right) .
$$

Step 2. Denote $A\left(N^{\circ}, M^{\circ}, L^{\circ}\right)=A_{N^{\circ}, M^{\circ}} \cdot A_{M^{\circ}, L^{\circ}} \cdot A_{N^{\circ}, L^{\circ}}^{-1}$. We will show that

$$
A\left(N^{\circ}, M^{\circ}, L^{\circ}\right)=C\left(N^{\circ}, M^{\circ}, L^{\circ}\right)^{-1} .
$$

Using formula (2.3) for the normalization coefficients, we can write $A\left(N^{\circ}, M^{\circ}, L^{\circ}\right)$ in the form

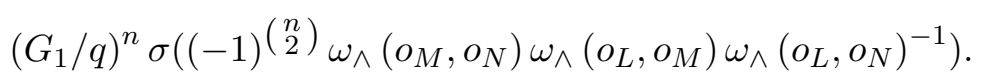

Lemma A.2. We have

$$
\omega_{\wedge}\left(r_{\wedge}^{L}\left(o_{M}\right), o_{M}\right)=(-1)^{n} \omega_{\wedge}\left(o_{M}, o_{N}\right) \omega_{\wedge}\left(o_{L}, o_{M}\right) \omega_{\wedge}\left(o_{L}, o_{N}\right)^{-1} .
$$

Using Lemma A.2, we get that $A\left(N^{\circ}, M^{\circ}, L^{\circ}\right)$ is equal to

$$
\left(G_{1} / q\right)^{n} \sigma\left((-1)^{\left(\begin{array}{c}
n \\
2
\end{array}\right)+n} \omega_{\wedge}\left(r_{\wedge}^{L}\left(o_{M}\right), o_{M}\right)\right)=C\left(N^{\circ}, M^{\circ}, L^{\circ}\right)^{-1},
$$

where in the second equality we use the identity $G_{1}^{2 n}=q^{n} \sigma\left((-1)^{n}\right)$.

This concludes the proof of the proposition. 
A.1.1. Proof of Lemma A.1. Choose an isomorphism $\varphi: M \stackrel{\sim}{\rightarrow} \mathbb{F}_{q}^{n}$. Let $B: M \times M \rightarrow \mathbb{F}_{q}$ be the symmetric form defined by $B=\varphi^{*}\left(\sum x_{i} y_{i}\right)$. Present the symmetric form $\omega\left(r^{L}(-),-\right)$ as

$$
\omega\left(r^{L}(-),-\right)=B(A(-),-),
$$

where $A \in \operatorname{Mat}_{n \times n}\left(\mathbb{F}_{q}\right)$ is a symmetric matrix. By definition $d\left[\omega\left(r^{L}(-)\right.\right.$, $-)]=\operatorname{det} A \bmod \mathbb{F}_{q}^{\times 2}$.

Let $e_{1}, e_{2}, \ldots, e_{n}$ be an orthonormal basis with respect to the form $B$. The argument now follows from

$$
\begin{aligned}
\operatorname{det} A & =\sum_{\sigma \in \Sigma_{n}}(-1)^{\sigma} \prod_{i=1}^{n} \omega\left(r^{L}\left(e_{i}\right), e_{\sigma(i)}\right) \\
& =(-1)^{\left(\begin{array}{c}
n \\
2
\end{array}\right)} \omega_{\wedge}\left(r_{\wedge}^{L}\left(e_{1} \wedge \cdots \wedge e_{n}\right), e_{1} \wedge \cdots \wedge e_{n}\right) \\
& =(-1)^{\left(\begin{array}{c}
n \\
2
\end{array}\right)} \omega_{\wedge}\left(r^{L}\left(o_{M}\right), o_{M}\right) \bmod \mathbb{F}_{q}^{\times 2} .
\end{aligned}
$$

This concludes the proof of the lemma.

A.1.2. Proof of Lemma A.2. We can write $r_{\wedge}^{L}\left(o_{M}\right)=a \cdot o_{N}$, for some $a \in \mathbb{F}_{q}$. The constant $a$ can be computed as follows: on the one hand, since $r^{L}(m)-m \in L$, we have that $\omega_{\wedge}\left(r_{\wedge}^{L}\left(o_{M}\right), o_{L}\right)=\omega_{\wedge}\left(o_{M}, o_{L}\right)$. On the other hand, we have that $\omega_{\wedge}\left(r_{\wedge}^{L}\left(o_{M}\right), o_{L}\right)=a \omega_{\wedge}\left(o_{N}, o_{L}\right)$, hence

$$
a=\frac{\omega_{\wedge}\left(o_{M}, o_{L}\right)}{\omega_{\wedge}\left(o_{N}, o_{L}\right)} .
$$

Therefore we obtain that

$$
\begin{aligned}
\omega_{\wedge}\left(r_{\wedge}^{L}\left(o_{M}\right), o_{M}\right) & =\omega_{\wedge}\left(o_{M}, o_{L}\right) \omega_{\wedge}\left(o_{N}, o_{L}\right)^{-1} \omega_{\wedge}\left(o_{N}, o_{M}\right) \\
& =(-1)^{n} \omega_{\wedge}\left(o_{M}, o_{N}\right) \omega_{\wedge}\left(o_{L}, o_{M}\right) \omega_{\wedge}\left(o_{L}, o_{N}\right)^{-1},
\end{aligned}
$$

where in the second equality we used the relation $\omega_{\wedge}\left(o, o^{\prime}\right)=(-1)^{n} \omega_{\wedge}\left(o^{\prime}, o\right)$. This concludes the proof of the lemma.

A.2. Proof of Proposition 2.7. The trivialization of the vector bundle $\mathfrak{H}$ is constructed as follows: Let $\left(N^{\circ}, L^{\circ}\right) \in \operatorname{OLag}(V)^{2}$. In order to construct $T_{N^{\circ}, L^{\circ}}$, choose a third $M^{\circ} \in \mathrm{OLag}(V)$ such that $\left(N^{\circ}, M^{\circ}\right),\left(M^{\circ}, L^{\circ}\right) \in U_{2}$ (such a choice always exists). Define

$$
T_{N^{\circ}, L^{\circ}}=T_{N^{\circ}, M^{\circ} \circ} T_{M^{\circ}, L^{\circ}}
$$

where we note that both operators in the composition on the left-hand side are well defined. We are left to show that the definition of $T_{N^{\circ}, L^{\circ}}$ does not depends on the choice of $M^{\circ}$. Let $M_{i}^{\circ} \in \operatorname{OLag}(V), i=1,2$, such that $\left(N^{\circ}, M_{i}^{\circ}\right),\left(M_{i}^{\circ}, L^{\circ}\right) \in U_{2}$. We want to show that

$$
T_{N^{\circ}, M_{1}^{\circ}} \circ T_{M_{1}^{\circ}, L^{\circ}}=T_{N^{\circ}, M_{2}^{\circ}} \circ T_{M_{2}^{\circ}, L^{\circ}} .
$$


Choose $M_{3}^{\circ} \in \operatorname{OLag}(V)$ such that $\left(M_{3}^{\circ}, M_{i}^{\circ}\right) \in U_{2}$ for $i=1,2$ and, in addition, $\left(M_{3}^{\circ}, L^{\circ}\right),\left(N^{\circ}, M_{3}^{\circ}\right) \in U_{2}$. We have

$$
\begin{aligned}
T_{N^{\circ}, M_{1}^{\circ}} \circ T_{M_{1}^{\circ}, L^{\circ}} & =T_{N^{\circ}, M_{1}^{\circ}} \circ T_{M_{1}^{\circ}, M_{3}^{\circ}} \circ T_{M_{3}^{\circ}, L^{\circ}} \\
& =T_{N^{\circ}, M_{3}^{\circ}} \circ T_{M_{3}^{\circ}, L^{\circ}},
\end{aligned}
$$

where the first and second equalities are the multiplicativity property for triples which are in general position pairwisely (Proposition 2.6). In the same fashion, we show that $T_{N^{\circ}, M_{2}^{\circ}} \circ T_{M_{2}^{\circ}, L^{\circ}}=T_{N^{\circ}, M_{3}^{\circ}} \circ T_{M_{3}^{\circ}, L^{\circ}}$.

Finally, the same kind of argument shows that the complete system $\left\{T_{M^{\circ}, L^{\circ}}:\left(M^{\circ}, L^{\circ}\right) \in \mathrm{OLag}(V)^{2}\right\}$ forms a trivialization.

This concludes the proof of the proposition.

A.3. Proof of Proposition 2.8. Denote $I=M \cap L$. Using the canonical decompositions $\bigwedge^{\text {top }} M=\bigwedge^{\text {top }} I \otimes \bigwedge^{\text {top }} M / I$ and $\bigwedge^{\text {top }} L=\bigwedge^{\text {top }} I \otimes$ $\bigwedge^{\text {top }} L / I$, we can write the orientations on $M$ and $L$ in the form $o_{M}=$ $\iota_{M} \otimes o_{M / I}$ and $o_{L}=\iota_{L} \otimes o_{L / I}$, respectively.

Choose a third $S^{\circ} \in \operatorname{OLag}(V)$ such that $\left(M^{\circ}, S^{\circ}\right),\left(S^{\circ}, L^{\circ}\right) \in U_{2}$. By Theorem 2.7, we conclude that $T_{M^{\circ}, L^{\circ}}=T_{M^{\circ}, S^{\circ}} \circ T_{S^{\circ}, L^{\circ}}$, furthermore, using the explicit formula $(2.2)$, we can write the composition $T_{M^{\circ}, S^{\circ}} \circ T_{S^{\circ}, L^{\circ}}$ explicitly in the form

$$
\left(G_{1} / q\right)^{2 n} \sigma\left(\omega_{\wedge}\left(o_{S}, o_{M}\right) \omega_{\wedge}\left(o_{L}, o_{S}\right)\right) F_{M^{\circ}, S^{\circ}} \circ F_{S^{\circ}, L^{\circ}} .
$$

Direct computation reveals that

$$
\begin{aligned}
F_{M^{\circ}, S^{\circ} \circ F_{S^{\circ}, L^{\circ}}} & =\left[\sum_{m \in M} \psi\left(\frac{1}{2} \omega\left(m, r^{L}(m)\right)\right)\right] \cdot F_{M^{\circ}, L^{\circ}} \\
& =\left[\# I \cdot \sum_{\bar{m} \in M / I} \psi\left(\frac{1}{2} \omega\left(\bar{m}, r^{L}(\bar{m})\right)\right)\right] \cdot F_{M^{\circ}, L^{\circ}},
\end{aligned}
$$

where $r^{L}: M \rightarrow S$ is the linear map defined by the condition $r^{L}(m)-m \in L$, for every $m \in M$; moreover, $\operatorname{ker}\left(r^{L}\right)=I$, hence, $r^{L}$ factors to an injective map $r^{L}: M / I \rightarrow S$. This also explains the second equality in (A.3).

Let us denote by $B: M / I \times M / I \rightarrow \mathbb{F}_{q}$ the symmetric form on $M / I$ given by $B\left(\bar{m}_{1}, \bar{m}_{2}\right)=\frac{1}{2} \omega\left(\bar{m}_{1}, r^{L}\left(\bar{m}_{2}\right)\right)$. An easy diagonalization argument implies that

$$
\sum_{\bar{m} \in M / I} \psi\left(\frac{1}{2} \omega\left(\bar{m}, r^{L}(\bar{m})\right)\right)=G_{1}^{n_{I}} \cdot \sigma(d[B]),
$$

where $d[B] \in \mathbb{F}_{q}^{\times} / \mathbb{F}_{q}^{\times 2}$ denotes the discriminant of the symmetric form $B$.

Let us denote by $B_{\wedge}$ the form on $\bigwedge^{\text {top }} M / I$ induce from $B$.

Lemma A.3. We have

$$
d[B]=(-1)^{\left(\begin{array}{c}
n_{I} \\
2
\end{array}\right)} B_{\wedge}\left(o_{M / I}, o_{M / I}\right) .
$$


QUANTIZATION OF SYMPLECTIC VECTOR SPACES OVER FINITE FIELDS 495

Summarizing, we obtain that $T_{M^{\circ}, S^{\circ}} \circ T_{S^{\circ}, L^{\circ}}$ is equal to

$$
\left(G_{1} / q\right)^{n_{I}} \sigma\left((-1)^{\left(\begin{array}{c}
n_{I} \\
2
\end{array}\right)+n} B_{\wedge}\left(o_{M / I}, o_{M / I}\right) \omega_{\wedge}\left(o_{S}, o_{M}\right) \omega_{\wedge}\left(o_{L}, o_{S}\right)\right) F_{M^{\circ}, L^{\circ}} .
$$

Finally use

Lemma A.4. We have

$$
B_{\wedge}\left(o_{M / I}, o_{M / I}\right)=(-1)^{n} \omega_{\wedge}\left(o_{L / I}, o_{M / I}\right) \omega_{\wedge}\left(o_{S}, o_{M}\right) \omega\left(o_{L}, o_{S}\right)^{-1} \frac{\iota_{L}}{\iota_{M}} .
$$

So we see that $T_{M^{\circ}, S^{\circ}} \circ T_{S^{\circ}, L^{\circ}}$ is equal to

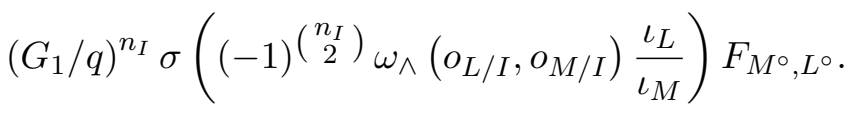

This concludes the proof of the proposition.

A.3.1. Proof of Lemma A.3. Choose an isomorphism $\varphi: M / I \stackrel{\widetilde{\Im}}{\rightarrow} \mathbb{F}_{q}^{n_{I}}$. Let $B_{0}: M / I \times M / I \rightarrow \mathbb{F}_{q}$ be the symmetric form defined by $B_{0}=$ $\varphi^{*}\left(\sum x_{i} y_{i}\right)$. Present the symmetric form $B$ as

$$
B(-,-)=B_{0}(A(-),-),
$$

where $A \in \operatorname{Mat}_{n_{I} \times n_{I}}\left(\mathbb{F}_{q}\right)$ is a symmetric matrix. By definition $d[B]=$ $\operatorname{det} A \bmod \mathbb{F}_{q}^{\times 2}$.

Let $e_{1}, e_{2}, \ldots, e_{n_{I}}$ be an orthonormal basis with respect to the form $B_{0}$. The argument follows from the following equalities:

$$
\begin{aligned}
\operatorname{det} A & =\sum_{\sigma \in \Sigma_{n_{I}}}(-1)^{\sigma} \prod_{i=1}^{n_{I}} \omega\left(e_{i}, r^{L}\left(e_{\sigma(i)}\right)\right) \\
& =(-1)^{\frac{n_{I}\left(n_{I}-1\right)}{2}} \omega_{\wedge}\left(e_{1} \wedge \cdots \wedge e_{n_{I}}, r_{\wedge}^{L}\left(e_{1} \wedge \cdots \wedge e_{n_{I}}\right)\right) \\
& =(-1)^{\frac{n_{I}\left(n_{I}-1\right)}{2}} \omega_{\wedge}\left(o_{M / I}, r^{L}\left(o_{M / I}\right)\right) \bmod \mathbb{F}_{q}^{\times 2} .
\end{aligned}
$$

This concludes the proof of the lemma.

A.3.2. Proof of Lemma A.4. Let us denote by $r_{\wedge}^{L}: \bigwedge^{\text {top }} M / I \rightarrow$ $\bigwedge^{\text {top }} r^{L}(M / I)$ the map induced from $r^{L}: M / I \rightarrow r^{L}(M / I)$. There is a canonical decomposition $\bigwedge^{\text {top }} S=\bigwedge^{\text {top }} r^{L}(M / I) \otimes \bigwedge^{\text {top }} S / r^{L}(M / I)$ and consequently the orientation $o_{S}$ can be written in the form $o_{S}=r_{\wedge}^{L}\left(o_{M / I}\right) \otimes$ o. On the one hand,

$$
\begin{aligned}
\omega_{\wedge}\left(o_{M}, o_{S}\right) & =\omega_{\wedge}\left(\iota_{M} \otimes o_{M / I}, r_{\wedge}^{L}\left(o_{M / I}\right) \otimes o\right) \\
& =\omega_{\wedge}\left(\iota_{M}, o\right) \cdot \omega_{\wedge}\left(o_{M / I}, r_{\wedge}^{L}\left(o_{M / I}\right)\right) .
\end{aligned}
$$


On the other hand,

$$
\begin{aligned}
\omega_{\wedge}\left(o_{L}, o_{S}\right) & =\omega_{\wedge}\left(\iota_{L} \otimes o_{L / I}, r_{\wedge}^{L}\left(o_{M / I}\right) \otimes o\right) \\
& =\omega_{\wedge}\left(\iota_{L}, o\right) \cdot \omega_{\wedge}\left(o_{L / I}, r_{\wedge}^{L}\left(o_{M / I}\right)\right) \\
& =\frac{\iota_{L}}{\iota_{M}} \omega_{\wedge}\left(\iota_{M}, o\right) \cdot \omega_{\wedge}\left(o_{L / I}, o_{M / I}\right)
\end{aligned}
$$

which implies that

$$
\omega_{\wedge}\left(\iota_{M}, o\right)=\omega_{\wedge}\left(o_{L}, o_{S}\right) \omega_{\wedge}\left(o_{L / I}, o_{M / I}\right)^{-1} \frac{\iota_{M}}{\iota_{L}} .
$$

Altogether, we obtain that

$\omega_{\wedge}\left(o_{M / I}, r_{\wedge}^{L}\left(o_{M / I}\right)\right)=(-1)^{N} \omega_{\wedge}\left(o_{L / I}, o_{M / I}\right) \omega_{\wedge}\left(o_{S}, o_{M}\right) \omega_{\wedge}\left(o_{L}, o_{S}\right)^{-1} \frac{\iota_{L}}{\iota_{M}}$.

This concludes the proof of the lemma.

A.4. Proof of Proposition 2.12. Let $f \in \operatorname{Mor}_{S y m p}\left(V_{1}, V_{2}\right)$ be an isomorphism of symplectic vector spaces. The map $f$ induces a pair isomorphisms

$$
\begin{aligned}
& r: H\left(V_{1}\right) \stackrel{\simeq}{\longrightarrow} H\left(V_{2}\right), \\
& s: \operatorname{OLag}\left(V_{1}\right) \stackrel{\simeq}{\longrightarrow} \operatorname{OLag}\left(V_{2}\right) .
\end{aligned}
$$

The first is the isomorphism of groups given by $r\left(v_{1}, z\right)=\left(f\left(v_{1}\right), z\right)$ and the second is the evident-induced bijection of sets. For every $L^{\circ} \in \operatorname{OLag}\left(V_{1}\right)$, we have a pull-back isomorphism $r_{L^{\circ}}^{*}: \mathcal{H}_{s\left(L^{\circ}\right)}\left(V_{2}\right) \stackrel{\widetilde{\sim}}{\rightarrow} \mathcal{H}_{L^{\circ}}\left(V_{1}\right)$.

Lemma A.5. For every $\left(M^{\circ}, L^{\circ}\right) \in O \operatorname{Lag}\left(V_{1}\right)^{2}$

$$
T_{M^{\circ}, L^{\circ}} \circ r_{L^{\circ}}^{*}=r_{M^{\circ}}^{*} \circ T_{s\left(M^{\circ}\right), s\left(L^{\circ}\right)} .
$$

The system of isomorphisms $\left\{r_{L^{\circ}}^{*}\right\}$ yields the required isomorphism of vector spaces $\mathcal{H}(f): \mathcal{H}\left(V_{2}\right) \stackrel{\widetilde{ }}{\rightarrow} \mathcal{H}\left(V_{1}\right)$; evidently, for every $f \in$ $\operatorname{Mor}_{\text {Symp }}\left(V_{1}, V_{2}\right), g \in \operatorname{Mor}_{\text {Symp }}\left(V_{2}, V_{3}\right)$

$$
\mathcal{H}(g \circ f)=\mathcal{H}(g) \circ \mathcal{H}(f) .
$$

This concludes the proof of the proposition.

A.4.1. Proof of Lemma A.5. It will be convenient to work with the system of kernels $\left\{K_{M^{\circ}, L^{\circ}}\right\}$ (Theorem 2.10). Recall, $T_{M^{\circ}, L^{\circ}}=I\left[K_{M^{\circ}, L^{\circ}}\right]$. It is enough to show

$$
r^{*} K_{s\left(M^{\circ}\right), s\left(L^{\circ}\right)}=K_{M^{\circ}, L^{\circ}},
$$

for every $\left(M^{\circ}, L^{\circ}\right) \in \mathrm{OLag}\left(V_{1}\right)^{2}$. Direct verification reveals that (A.5) holds when $\left(M^{\circ}, L^{\circ}\right) \in U_{2}$. Given $\left(M^{\circ}, L^{\circ}\right) \in \operatorname{OLag}\left(V_{1}\right)^{2}$, let $S^{\circ} \in \operatorname{OLag}\left(V_{1}\right)$ be an oriented Lagrangian such that $\left(M^{\circ}, S^{\circ}\right),\left(S^{\circ}, L^{\circ}\right) \in U_{2}$. Write

$$
\begin{aligned}
r^{*} K_{s\left(M^{\circ}\right), s\left(L^{\circ}\right)} & =r^{*}\left(K_{s\left(M^{\circ}\right), s\left(S^{\circ}\right)} * K_{s\left(S^{\circ}\right), s\left(L^{\circ}\right)}\right) \\
& =r^{*} K_{s\left(M^{\circ}\right), s\left(S^{\circ}\right)} * r^{*} K_{s\left(S^{\circ}\right), s\left(L^{\circ}\right)} \\
& =K_{M^{\circ}, S^{\circ}} * K_{S^{\circ}, L^{\circ}}=K_{M^{\circ}, L^{\circ}},
\end{aligned}
$$


where the first and fourth equalities are by the multiplicativity property of the system $\left\{K_{M^{\circ}, L^{\circ}}\right\}$ (see Theorem 2.10) and the second equality follows from the fact that $r$ is a morphism of groups.

This concludes the proof of the lemma.

A.5. Proof of Proposition 2.14. Let $V_{i} \in$ Symp, $i=1,2$. There are two maps

$$
\begin{aligned}
& r: H\left(V_{1}\right) \times H\left(V_{2}\right) \longrightarrow H\left(V_{1} \times V_{2}\right), \\
& s: \operatorname{OLag}\left(V_{1}\right) \times \operatorname{OLag}\left(V_{1}\right) \longrightarrow \operatorname{OLag}\left(V_{1} \times V_{2}\right) .
\end{aligned}
$$

The map $r$ is a surjective morphism of groups given by $r\left(\left(v_{1}, z_{1}\right)\right.$, $\left.\left(v_{2}, z_{2}\right)\right)=\left(\left(v_{1}, v_{2}\right), z_{1}+z_{2}\right)$. The map $s$ is a bijection of sets given by

$$
s\left(L_{1}^{\circ}, L_{2}^{\circ}\right)=\left(L_{1} \times L_{2}, o_{L_{1}} \otimes o_{L_{2}}\right),
$$

for $L_{i}=\left(L_{i}, o_{L_{i}}\right), i=1,2$, where we use the canonical isomorphism of vector spaces

$$
\bigwedge^{\text {top }}\left(L_{1} \times L_{2}\right) \simeq \bigwedge^{\text {top }} L_{1} \otimes \bigwedge^{\text {top }} L_{2}
$$

For every $\left(L_{1}^{\circ}, L_{2}^{\circ}\right) \in \operatorname{OLag}\left(V_{1}\right) \times \operatorname{OLag}\left(V_{1}\right)$, the map $r$ induces a pull back isomorphism $r_{\left(L_{1}^{\circ}, L_{2}^{\circ}\right)}^{*}: \mathcal{H}_{s\left(L_{1}^{\circ}, L_{2}^{\circ}\right)} \stackrel{\simeq}{\rightarrow} \mathcal{H}_{L_{1}^{\circ}} \otimes \mathcal{H}_{L_{2}^{\circ}}$.

The proof of the following lemma follows along similar lines as the proof of Lemma A.5.

Lemma A.6. For every $\left(L_{1}^{\circ}, L_{2}^{\circ}\right),\left(M_{1}^{\circ}, M_{2}^{\circ}\right) \in \operatorname{OLag}\left(V_{1}\right) \times \operatorname{OLag}\left(V_{1}\right)$

$$
T_{M_{1}^{\circ}, L_{1}^{\circ}} \otimes T_{M_{2}^{\circ}, L_{2}^{\circ}} \circ r_{\left(L_{1}^{\circ}, L_{2}^{\circ}\right)}^{*}=r_{\left(M_{1}^{\circ}, M_{2}^{\circ}\right)}^{*} \circ T_{s\left(M_{1}^{\circ}, M_{2}^{\circ}\right), s\left(L_{1}^{\circ}, L_{2}^{\circ}\right)} \cdot
$$

The system $\left\{r_{\left(L_{1}^{\circ}, L_{2}^{\circ}\right)}^{*}\right\}$ gives the required isomorphism $\alpha_{V_{1} \times V_{2}}$.

This concludes the proof of the proposition.

A.6. Proof of Proposition 2.16. Let $r: H(V) \rightarrow H(\bar{V})$ be the isomorphism of Heisenberg groups, given by $r(v, z)=(v,-z)$. For every oriented Lagrangian $L^{\circ} \in \mathrm{OLag}(V)=\mathrm{OLag}(\bar{V})$, the map $r$ induces a the pull-back isomorphism

$$
r_{L^{\circ}}^{*}: \mathcal{H}_{L^{\circ}}(\bar{V}, \psi) \stackrel{\simeq}{\rightarrow} \mathcal{H}_{L^{\circ}}\left(V, \psi^{-1}\right) .
$$

We denote by $\bar{T}_{M^{\circ}, L^{\circ}}$ and $T_{M^{\circ}, L^{\circ}}$ the trivializations of $\mathfrak{H}\left(\bar{V}, \psi^{-1}\right)$ and $\mathfrak{H}(V, \psi)$, respectively.

Lemma A.7. For every $\left(M^{\circ}, L^{\circ}\right) \in \operatorname{OLag}(V)^{2}$

$$
r_{M^{\circ}}^{*} \circ \bar{T}_{M^{\circ}, L^{\circ}}=T_{M^{\circ}, L^{\circ}} \circ r_{L^{\circ}}^{*} .
$$


The system $\left\{r_{L^{\circ}}^{*}\right\}$ gives an isomorphism $r^{*}: \mathcal{H}(\bar{V}, \psi) \stackrel{\widetilde{ }}{\rightarrow} \mathcal{H}\left(V, \psi^{-1}\right)$. Finally, there is a natural non-degenerate pairing

$$
\langle\cdot, \cdot\rangle_{V}^{\prime}: \mathcal{H}\left(V, \psi^{-1}\right) \times \mathcal{H}(V, \psi) \longrightarrow \mathbb{C},
$$

induced from the system pairings $\langle\cdot, \cdot\rangle_{L^{\circ}}^{\prime}: \mathcal{H}_{L^{\circ}}\left(V, \psi^{-1}\right) \times \mathcal{H}_{L^{\circ}}(V, \psi) \rightarrow \mathbb{C}$, given by

$$
\langle f, g\rangle_{L^{\circ}}^{\prime}=\sum_{v \in V / L} g(v) f(v),
$$

for every $f \in \mathcal{H}_{L^{\circ}}\left(V, \psi^{-1}\right)$ and $g \in \mathcal{H}_{L^{\circ}}(V, \psi)$; we note that the function $g \cdot f: H(V) \rightarrow \mathbb{C}$ descends to a function on $V / L$. Define

$$
\langle\cdot, \cdot\rangle_{V}=\left\langle r^{*}(\cdot), \cdot\right\rangle_{V}^{\prime} \cdot
$$

This concludes the proof of the proposition.

A.6.1. Proof of Lemma A.7. It is enough to prove the condition for $\left(M^{\circ}, L^{\circ}\right) \in U_{2}$. Let $f \in \mathcal{H}_{L^{\circ}}(\bar{V}, \psi)$. On the one hand,

$$
\begin{aligned}
r_{M^{\circ}}^{*} \bar{T}_{M^{\circ}, L^{\circ}}[f](v, z) & =\bar{T}_{M^{\circ}, L^{\circ}}[f](v,-z) \\
& =\bar{A}_{M^{\circ}, L^{\circ}} \sum_{m \in M} f\left(m+v,-z-\frac{1}{2} \omega(m, v)\right) .
\end{aligned}
$$

On the other hand,

$$
\begin{aligned}
T_{M^{\circ}, L^{\circ}}\left[r_{L^{\circ}}^{*}(f)\right](v, z) & =A_{M^{\circ}, L^{\circ}} \sum_{m \in M} r_{L^{\circ}}^{*}(f)\left(m+v, z+\frac{1}{2} \omega(m, v)\right) \\
& =A_{M^{\circ}, L^{\circ}} \sum_{m \in M} f\left(m+v,-z-\frac{1}{2} \omega(m, v)\right) .
\end{aligned}
$$

Noting that $\bar{A}_{M^{\circ}, L^{\circ}}=A_{M^{\circ}, L^{\circ}}$, the result follows.

This concludes the proof of the lemma.

A.7. Proof of Proposition 2.18. Let $V \in$ Symp. Let $I^{\circ}=\left(I, o_{I}\right)$ be an oriented isotropic subspace in $V$. There is a map $s: \operatorname{OLag}\left(I^{\perp} / I\right) \longrightarrow$ $\operatorname{OLag}(V)$, given by

$$
s\left(L, o_{L}\right)=\left(\mathrm{pr}^{-1}(L), o_{I} \otimes o_{L}\right),
$$

for $\left(L, o_{L}\right) \in \mathrm{OLag}\left(I^{\perp} / I\right)$, where pr $: I^{\perp} \rightarrow I^{\perp} / I$ is the natural projection and we use the canonical isomorphism

$$
\bigwedge^{\text {top }} \operatorname{pr}^{-1}(L) \simeq \bigwedge^{\text {top }} I \otimes \bigwedge^{\text {top }} L
$$

For every $L^{\circ} \in \operatorname{OLag}\left(I^{\perp} / I\right)$ there is a "pull-back" map $r_{L^{\circ}}^{*}: \mathcal{H}_{s\left(L^{\circ}\right)}(V) \rightarrow$ $\mathcal{H}_{L^{\circ}}\left(I^{\perp} / I\right)$ defined as follows: given $f \in \mathcal{H}_{s\left(L^{\circ}\right)}(V)$, the function $r_{L^{\circ}}^{*}(f)$ is 
the restriction $f_{\mid Z \cdot I^{\perp}}$, which descends to a function on $H\left(I^{\perp} / I\right)$. It is easy to verify that $r_{L^{\circ}}^{*}$ maps $\mathcal{H}_{s\left(L^{\circ}\right)}(V)^{I}$ isomorphically onto $\mathcal{H}_{L^{\circ}}\left(I^{\perp} / I\right)$.

Lemma A.8. For every $\left(M^{\circ}, L^{\circ}\right) \in \mathrm{OLag}\left(I^{\perp} / I\right)$

$$
F_{M^{\circ}, L^{\circ}} \circ r_{L^{\circ}}^{*}=r_{M^{\circ}}^{*} \circ F_{s\left(M^{\circ}\right), s\left(L^{\circ}\right)} .
$$

The system $\left\{r_{L^{\circ}}^{*}\right\}$ gives the required isomorphism $\alpha_{\left(I^{\circ}, V\right)}: \mathcal{H}(V)^{I} \stackrel{\widetilde{\rightrightarrows}}{\rightarrow}$ $\mathcal{H}\left(I^{\perp} / I\right)$.

This concludes the proof of the proposition.

A.7.1. Proof of Lemma A.8. It will be convenient to work with the canonical system of kernels $\left\{K_{M^{\circ}, L^{\circ}}\right\}$ (Proposition 2.10), recall we have $F_{M^{\circ}, L^{\circ}}=I\left[K_{M^{\circ}, L^{\circ}}\right]$. Let pr $: I^{\perp} \rightarrow I^{\perp} / I$ be the canonical projection and let $i: I^{\perp} \rightarrow V$ denote the inclusion. It is enough to show that

$$
i^{*} K_{s\left(M^{\circ}\right), s\left(L^{\circ}\right)}=\operatorname{pr}^{*} K_{M^{\circ}, L^{\circ}},
$$

for every $\left(M^{\circ}, L^{\circ}\right) \in O \operatorname{Lag}\left(I^{\perp} / I\right)^{2}$. This is done by direct verification using the formulas from Proposition 2.8. This concludes the proof of the lemma.

A.8. Proof of Lemma 3.4. The proof will proceed in several steps.

Step 1. First, we prove that the sheaf $p_{12}^{*} \mathcal{K} * p_{23}^{*} \mathcal{K}$ is irreducible $\left[n_{3}\right]$ perverse on $\mathbf{U}_{3} \times \mathbf{H}(\mathbf{V})$ and for this, it is enough to show that $p_{12}^{*} \tilde{\mathcal{K}} * p_{23}^{*} \tilde{\mathcal{K}}$ is irreducible $\left[n_{3}\right]$-perverse on $\mathbf{U}_{3} \times \mathbf{H}(\mathbf{V})$.

The convolution $p_{12}^{*} \tilde{\mathcal{K}} * p_{23}^{*} \tilde{\mathcal{K}}$ can be written explicitly as

$$
p_{12}^{*} \tilde{\mathcal{K}} * p_{23}^{*} \tilde{\mathcal{K}}\left(N^{\circ}, M^{\circ}, L^{\circ}, h\right) \simeq \int_{n \in \mathbf{N}} \tilde{\mathcal{K}}\left(N^{\circ}, M^{\circ}, h \cdot n^{-1}\right) \otimes \tilde{\mathcal{K}}\left(M^{\circ}, L^{\circ}, n\right) .
$$

It is enough to consider the case when $h=m \in \mathbf{M}$ where we get

$$
p_{12}^{*} \tilde{\mathcal{K}} * p_{23}^{*} \tilde{\mathcal{K}}\left(N^{\circ}, M^{\circ}, L^{\circ}, m\right) \simeq \int_{n \in \mathbf{N}} \mathcal{L}_{\psi}(\omega(n, m)) \otimes \tilde{\mathcal{K}}\left(M^{\circ}, L^{\circ}, n\right) .
$$

We see that $p_{12}^{*} \tilde{\mathcal{K}} * p_{23}^{*} \tilde{\mathcal{K}}\left(N^{\circ}, M^{\circ}, L^{\circ},-\right)_{\mid \mathbf{M}}$ is an application of $\ell$-adic Fourier transform to $\tilde{\mathcal{K}}\left(M^{\circ}, L^{\circ},-\right){ }_{\mid \mathbf{N}}$, hence, by the Katz-Laumon theorem $[\mathbf{1 6}] p_{12}^{*} \tilde{\mathcal{K}} * p_{23}^{*} \tilde{\mathcal{K}}$ is irreducible $\left[n_{3}\right]$-perverse.

Remark A.9. A more functorial way to describe the previous development is as follows: Let $\mathbf{C}_{i} \rightarrow \mathbf{U}_{3}, i=1,2,3$, denote the tautological vector bundle with fibers $\left(\mathbf{C}_{i}\right)_{\mid\left(L_{1}^{\circ}, L_{2}^{\circ}, L_{3}^{\circ}\right)}=\mathbf{L}_{i}$. What we showed is that

$$
\left(p_{12}^{*} \tilde{\mathcal{K}} * p_{23}^{*} \tilde{\mathcal{K}}\right)_{\mid \mathbf{C}_{2}} \simeq \operatorname{Four}_{\mathbf{C}_{2}, \mathbf{C}_{1}}\left(p_{23}^{*} \tilde{\mathcal{K}}_{\mid \mathbf{C}_{1}}\right)
$$

where Four $_{\mathbf{C}_{2}, \mathbf{C}_{1}}$ is the $\ell$-adic Fourier transform from $\mathrm{D}^{\mathrm{b}}\left(\mathbf{C}_{1}\right)$ to $D^{\mathrm{b}}\left(\mathbf{C}_{2}\right)$, induced from the pairing $w: \mathbf{C}_{1} \times \mathbf{U}_{3} \mathbf{C}_{2} \rightarrow \mathbb{G}_{\mathrm{a}, \mathbf{U}_{3}}$. 
Step 2. It is enough to show that the sheaves $p_{13}^{*} \mathcal{K}$ and $p_{12}^{*} \mathcal{K} * p_{23}^{*} \mathcal{K}$ are isomorphic on the zero section $\mathbf{U}_{3} \times\{0\} \subset \mathbf{U}_{3} \times \mathbf{H}(\mathbf{V})$. We have

$$
p_{12}^{*} \mathcal{K} * p_{23}^{*} \mathcal{K} \simeq p_{12}^{*} \mathcal{A} \otimes p_{23}^{*} \mathcal{A} \otimes p_{12}^{*} \tilde{\mathcal{K}}_{*} p_{23}^{*} \tilde{\mathcal{K}}
$$

Direct computation reveals that

$$
p_{12}^{*} \tilde{\mathcal{K}} * p_{23}^{*} \tilde{\mathcal{K}}\left(N^{\circ}, M^{\circ}, L^{\circ}, 0\right) \simeq \int_{n \in \mathbf{N}} \mathcal{L}_{\psi}\left(\frac{1}{2} \omega\left(r^{M}(n), n\right)\right),
$$

where $r^{M}: \mathbf{N} \rightarrow \mathbf{L}$ is the linear map defined by the relation $r^{M}(n)-n \in \mathbf{M}$ for every $n \in \mathbf{N}$. The map $r^{M}$ induces a map $r_{\wedge}^{M}: \bigwedge^{t o p} \mathbf{N} \rightarrow \bigwedge^{t o p} \mathbf{L}$. There exists an isomorphism

$$
\int_{n \in \mathbf{N}} \mathcal{L}_{\psi}\left(\frac{1}{2} \omega\left(r^{M}(n), n\right)\right) \simeq \mathcal{G}_{1}^{\otimes n} \otimes \mathcal{L}_{\sigma}\left((-1)^{\left(\begin{array}{l}
n \\
2
\end{array}\right)} \omega_{\wedge}\left(r_{\wedge}^{M}\left(o_{N}\right), o_{N}\right)\right) .
$$

Combining everything together we obtain

$$
\begin{aligned}
& p_{12}^{*} \mathcal{K} * p_{23}^{*} \mathcal{K}\left(N^{\circ}, M^{\circ}, L^{\circ}, 0\right) \\
& \simeq \mathcal{G}_{1}^{\otimes 3 n} \otimes \mathcal{L}_{\sigma}\left((-1)^{\left(\begin{array}{l}
n \\
2
\end{array}\right)} \omega_{\wedge}\left(o_{M}, o_{N}\right) \cdot \omega_{\wedge}\left(o_{L}, o_{M}\right)\right. \\
& \left.\left.\cdot \omega_{\wedge}\left(r_{\wedge}^{M}\left(o_{N}\right), o_{N}\right)\right)\right)[4 n](2 n) \\
& \simeq \mathcal{G}_{1}^{\otimes n} \otimes \mathcal{L}_{\sigma}\left((-1)^{\left(\begin{array}{c}
n \\
2
\end{array}\right)+n} \omega_{\wedge}\left(o_{M}, o_{N}\right)\right. \\
& \left.\left.\cdot \omega_{\wedge}\left(o_{L}, o_{M}\right) \cdot \omega_{\wedge}\left(r_{\wedge}^{M}\left(o_{N}\right), o_{N}\right)\right)\right)[2 n](n) \\
& \simeq \mathcal{G}_{1}^{\otimes n} \otimes \mathcal{L}_{\sigma}\left((-1)^{\left(\begin{array}{c}
n \\
2
\end{array}\right)} \omega_{\wedge}\left(o_{L}, o_{N}\right)\right)[2 n](n) \\
& \simeq p_{13}^{*} \mathcal{K}\left(N^{\circ}, M^{\circ}, L^{\circ}, 0\right) \text {. }
\end{aligned}
$$

where in the second isomorphism we used $\mathcal{G}_{1}^{\otimes 2 n} \simeq \mathcal{L}_{\sigma}\left((-1)^{n}\right)[-2 n](-n)$ and the third isomorphism follows from the identity

$$
\left.\omega_{\wedge}\left(r_{\wedge}^{M}\left(o_{N}\right), o_{N}\right)\right)=(-1)^{n} \omega_{\wedge}\left(o_{N}, o_{L}\right) \cdot \omega_{\wedge}\left(o_{M}, o_{N}\right) \omega_{\wedge}\left(o_{M}, o_{L}\right)^{-1},
$$

which holds by Lemma A.2.

This concludes the proof of Lemma 3.4.

A.9. Proof of Lemma 3.5. Lemma 3.4 implies that the sheaves $p_{13}^{*} \mathcal{K}$ and $p_{12}^{*} \mathcal{K} * p_{23}^{*} \mathcal{K}$ are isomorphic on the open subvariety $\mathbf{U}_{3} \times \mathbf{H}(\mathbf{V}) \subset \mathbf{V}_{3} \times \mathbf{H}(\mathbf{V})$. The sheaf $p_{13}^{*} \mathcal{K}$ is irreducible $\left[n_{3}\right]$-perverse as a pull-back by a smooth, surjective with connected fibers morphism, of an irreducible $\left[n_{2}\right]$-perverse sheaf on $\operatorname{OLag}(\mathbf{V})^{2} \times \mathbf{H}(\mathbf{V})$. Hence, it is enough to show that the sheaf $p_{12}^{*} \mathcal{K} * p_{23}^{*} \mathcal{K}$ irreducible $\left[n_{3}\right]$-perverse.

The last assertion follows from the fact that $p_{12}^{*} \mathcal{K} * p_{23}^{*} \mathcal{K}$ is principally an application of a, properly normalized, Fourier transform (see formula (A.6)) on $p_{23}^{*} \mathcal{K}$, hence by the Katz-Laumon theorem [16] it is irreducible $\left[n_{3}\right]$-perverse. 
Let us summarize. We showed that both sheaves $p_{13}^{*} \mathcal{K}$ and $p_{12}^{*} \mathcal{K} * p_{23}^{*} \mathcal{K}$ are irreducible $\left[n_{3}\right]$-perverse and are isomorphic on an open subvariety. This implies that they must be isomorphic. This concludes the proof of the lemma.

\section{References}

[1] J. Bernstein, Private communication, Max Planck Institute, Bonn, Germany, August, 2004.

[2] A. Beilinson, J. Bernstein and P. Deligne, Faisceaux pervers, Analysis and topology on singular spaces, I, Asterisque, 100, Soc. Math. France, Paris, 1982, 5-171.

[3] S. Bates and A. Weinstein, Lectures on the geometry of quantization, Berkeley Mathematics Lecture Notes, 8, American Mathematical Society, Providence, RI, 1997.

[4] P. Deligne, La conjecture de Weil II, Publ. Math. I.H.E.S 52 (1981), 313-428.

[5] O. Gabber, Private communication, 2006.

[6] D. Gaitsgory, Informal introduction to geometric Langlands, An introduction to the Langlands program, Jerusalem 2001, Birkhauser, Boston, MA, 2003, 269-281.

[7] P. Gérardin, Weil representations associated to finite fields, J. Algebra 46(1) (1977), 54-101.

[8] S. Gurevich and R. Hadani, Heisenberg realizations, eigenfunctions and proof of the Kurlberg-Rudnick supremum conjecture, arXiv:math-ph/0511036, 2005.

[9] S. Gurevich and R. Hadani, Notes on quantization of symplectic vector spaces over finite fields, in 'Proceedings of the EMS Summer School, Arithmetic and Geometry Around Quantization, Istanbul, June 5-15 2006' (O. Ceyhan, Yu. I. Manin, M. Marcolli, Max-Planck Institute, Bonn, Germany, eds.), Progress in Mathematics, Birkhauser, to appear (2009).

[10] A. Grothendieck, Formule de Lefschetz et rationalité des fonctions L, Seminaire Bourbaki 9 (1964), Exp. No. 279.

[11] V. Guillemin and S. Sternberg, Some problems in integral geometry and some related problems in microlocal analysis, Amer. J. Math. 101 (1979), 915-955.

[12] R. Hadani, The geometric Weil Representation and some applications, Ph.D. thesis, Tel Aviv University, 2006.

[13] R. Howe, On the character of Weil's representation, Trans. Amer. Math. Soc. 177 (1973), 287-298.

[14] D. Kazhdan, Private communication, Hebrew University, Jerusalem, Israel, March 2004.

[15] Kirillov, A. A. Unitary representations of nilpotent Lie groups, Uspehi Mat. Nauk 17 (1962), 57-110.

[16] N. M. Katz and G. Laumon, Transformation de Fouriér et majoration de sommes exponentièlles, Inst. Hautes Etudes Sci. Publ. Math. 62 (1985), 361-418.

[17] B. Kostant, Quantization and unitary representations, Lecture Notes in Math., 170, Springer-Verlag, 1970, 87-207.

[18] G. Lion, and M. Vergne, The Weil representation, Maslov index and theta series, Progress in Mathematics, 6, Birkhäuser, Boston, MA, 1980.

[19] A. Weil, Sur certains groupes d'operateurs unitaires, Acta Math. 111 (1964) 143-211.

[20] A. Weinstein, Symplectic geometry, Bull. Amer. Math. Soc. (N.S.) 5(1) (1981), 1-13. 
[21] A. Weinstein, The symplectic "category", Differential geometric methods in mathematical physics, Lecture Notes in Math., 905, Springer, Berlin-New York, 1982, 45-51.

Department of Mathematics

UNIVERSITY OF CALIFORNIA

Berkeley, CA 94720

E-mail address: shamgar@math.berkeley.edu

Department of Mathematics

UNIVERSITY OF CHICAGO

IL 60637

E-mail address: hadani@math.uchicago.edu

Received 09/12/2008, accepted 06/09/2009

We would like to thank our scientific advisor J. Bernstein for his interest and guidance, and for his idea about the notion of oriented Lagrangian subspace. It is a pleasure to thank D. Kazhdan for sharing with us his thoughts [14] about the possible existence of canonical Hilbert spaces. We thank A. Weinstein for several very influential discussions and for the opportunity to present this work in the symplectic geometry seminar, Berkeley, February 2007. We would like to acknowledge M. Vergne for the encouragement to write this paper. Finally, we would like to thank O. Ceyhan and the organizers of the conference AGAQ, held in Istanbul during June 2006, for the invitation to present this work. 